Mechatronics

Elsevier Editorial system(tm) for

Manuscript Draft

Manuscript Number:

Title: The Cross-Coupling of Lateral-Longitudinal Vehicle Dynamics: Towards Decentralized Fault-Tolerant Control Schemes

Article Type: SI: Auto and Intel Vehicles

Keywords: Fault tolerant control; fault detection; vehicle dynamics; coupling dynamics

Corresponding Author: Ms. Diana Hernandez-Alcantara,

Corresponding Author's Institution: ITESM

First Author: Diana Hernandez-Alcantara

Order of Authors: Diana Hernandez-Alcantara; Luis Amezquita-Brooks, Phd; Ruben Morales-Menendez, Phd; Olivier Sename, Phd; Luc Dugard, Phd

Abstract: In recent years there has been an increasing interest in improving vehicle characteristics through the use of Vehicle control Systems (VCS). In particular, VCS for the lateral (steering) and longitudinal (velocity) dynamics are used to improve the handling properties of a vehicle. Nonetheless, the introduction of the additional elements required for implementing these control systems also increases the possibility of faults. This problem can be mitigated by using Fault Tolerant Control(FTC) systems. The most common approach for steering FTC design is based on the use of a linear Bicycle Model (BM). Using this model decentralized steering controllers can be designed. However, the BM lacks significant lateral and longitudinal cross-coupling dynamics. In fact, the steering and velocity control problem could be viewed as a multivariable cross-coupled problem. In this article VCS for the steering and velocity are designed. The resulting controllers are decentralized and capable of practically eliminating the cross-coupling. A further problem, which has not been widely reported, is the propagation of the failure of one subsystem to other subsystems. It is shown that when the Velocity Control System (VelCS) fails, then the steering subsystem has a degraded performance due to cross-coupling. The main contribution of this article consists in showing that it is possible to detect and accommodate a failure of the VelcS within the steering control system, i.e. without requiring communication among subsystems. This enables a fully independent operation even if faults occur, that is a Decentralized Fault-Tolerant Control Scheme. 
December 27, 2016

Dear Editor of Mechatronics,

Dear Guest Editors of the special issue on Automated and Intelligent Vehicles,

It is a pleasure to submit the paper titled: "The Cross-Coupling of Lateral- Longitudinal Vehicle Dynamics: Towards Decentralized Fault-Tolerant Control Schemes" to this prestigious journal, hoping that this manuscript can be accepted for publication. We think that our paper is within the scope of the Special Issue "Automated and Intelligent Vehicles".

In recent years there has been an increasing interest in improving vehicle safety characteristics through the use of Fault Tolerant Control (FTC) schemes. In particular, FTC for the lateral (steering) and longitudinal (velocity) dynamics are relevant. In this context, decentralized schemes have attractive practical characteristics, but tend to have poor performance in highly cross-coupled systems. In this article the issue of developing decentralized FTC schemes for the longitudinal and lateral vehicle dynamics is addressed. The main contributions of the paper are:

a. In-depth study of the longitudinal-lateral dynamics cross-coupling.

b. Design of decentralized controllers for the longitudinal-lateral dynamics which are able to practically eliminate cross-coupling.

c. In-depth study of the effects of the propagation of failures in the longitudinal subsystem to the lateral subsystem.

d. Design of a decentralized FTC scheme which is able to accommodate a failure of the longitudinal subsystem within the lateral subsystem, without requiring communication between them.

We believe that these issues (particularly c and d) have not been fully addressed in current literature and will be of interest to the community.

Authors hope that this letter can explain the importance of this study and its contributions into the automotive control field. We confirm that this manuscript has not been published elsewhere and is not under consideration by another journal.

Sincerely yours,

Corresponding Author

Diana Hernandez-Alcantara, $\mathrm{PhD}$

School of Engineering and Sciences, Tecnológico de Monterrey Campus Monterrey 


\title{
The Cross-Coupling of Lateral-Longitudinal Vehicle Dynamics: Towards Decentralized Fault-Tolerant Control Schemes
}

\author{
Diana Hernandez-Alcantara ${ }^{\mathrm{a}}$, Luis Amezquita-Brooks ${ }^{\mathrm{b}}$, Ruben \\ Morales-Menendez $^{\mathrm{a}}$, Olivier Sename ${ }^{\mathrm{c}}$, Luc Dugard ${ }^{\mathrm{c}}$ \\ ${ }^{a}$ Tecnológico de Monterrey, School of Engineering and Sciences, Av. Garza Sada \# 2501 \\ 64849, Monterrey N.L., México (e-mail: \{d.hernandez.phd.mty,rmm\}@itesm.mx) \\ ${ }^{b}$ Universidad Autónoma de Nuevo León, Av. Universidad s/n. San Nicolás de los Garza, \\ N.L., México (luis.amezquitabrk@uanl.edu.mx) \\ ${ }^{c}$ GIPSA-Lab, Grenoble INP. Domaine Universitaire BP46, Saint Martin d'Hères, France \\ (\{olivier.sename, luc.dugard\}@gipsa-lab.grenoble-inp.fr)
}

\begin{abstract}
In recent years there has been an increasing interest in improving vehicle characteristics through the use of Vehicle Control Systems (VCS). In particular, VCS for the lateral (steering) and longitudinal (velocity) dynamics are used to improve the handling properties of a vehicle. Nonetheless, the introduction of the additional elements required for implementing these control systems also increases the possibility of faults. This problem can be mitigated by using Fault Tolerant Control (FTC) systems. The most common approach for steering FTC design is based on the use of a linear Bicycle Model $(B M)$. Using this model decentralized steering controllers can be designed. However, the $B M$ lacks significant lateral and longitudinal cross-coupling dynamics. In fact, the steering and velocity control problem could be viewed as a multivariable cross-coupled problem. In this article $V C S$ for the steering and velocity are designed. The resulting controllers are decentralized and capable of practically eliminating the cross-coupling. A further problem, which has not been widely reported, is the propagation of the failure of one subsystem to other subsystems. It is shown that when the Velocity Control System ( VelCS) fails, then the steering subsystem has a degraded performance due to cross-coupling. The main contribution of this article consists in showing that it is possible to detect and accommodate a failure of the VelCS within the steering control system, i.e. without requiring communication among
\end{abstract}


subsystems. This enables a fully independent operation even if faults occur, that is a Decentralized Fault Tolerant-Control Scheme.

Keywords: Fault tolerant control, fault detection, vehicle dynamics, coupling dynamics

\section{Introduction}

Passenger vehicles are complex systems made of several interconnected subsystems such as braking, suspension, steering, powertrain, etc. The recent technological development in (micro)-electronics, actuators and sensors has enabled the use of a wide diversity of Vehicle Control Systems (VCS) such as the Anti-lock Braking System $(A B S)$ and the Electronic Stability Control $(E S C)$. These systems allow the vehicle to comply with increasingly stringent requirements for efficiency, safety and handling qualities.

The handling properties of the vehicle can be described fundamentally by the lateral and longitudinal dynamics. The lateral dynamics mainly deal with the steering behavior whereas the longitudinal dynamics mainly deal with the vehicle velocity. Due to the recent interest in controlling the vehicle movement, the design of improved $V C S$ for these vehicle subsystems has gained attention.

Fault Tolerant Control (FTC) is also of particular interest as failures of any $V C S$ may degrade the overall handling capabilities and safety. Many model-based approaches for $F T C$ design have been proposed in literature, [1]. Their success depends on model accuracy; however, the most accurate vehicle models can be complex due to the nonlinearities and cross-coupling among subsystems.

Since each $V C S$ focuses in a particular vehicle subsystem, it is common to use simplified models which facilitate the design. That is, a decentralized control design approach is often used, in which each subsystem is treated independently. This approach also has the advantage of being easy to implement and introduces the possibility of implementing control systems that are physically independent.

The simplest model for steering $F T C$ design is the well-known linear Bicycle Model (BM). In [2] the authors developed a fault tolerant monitoring system based on a state observer to detect and isolate sensor faults. Using the same model, in [3] a sensor FTC strategy based on a switching Kalman Filter was proposed. In this case the FTC used the estimated state instead of the 
sensor measurements when faults occur. The drawback of these approaches is that they rely on the validity of the $B M$, which is a simplification of the lateral dynamics that neglects the suspension, load transfer and velocity dynamics.

This shortcoming can be partially alleviated by using more comprehensive vehicle models. In [4] a Fault Detection and Isolation (FDI) method based on structured residuals was developed for sensor and actuator faults in an over-actuated $X$-by-Wire vehicle. In this case the authors used an extended $B M$ which also considers the longitudinal dynamics. In [5] the authors used a Takagi-Sugeno $(T-S)$ fuzzy $B M$ to take into account the nonlinear tire behavior and proposed a proportional integral observer to estimate actuator faults. By using the T-S model, in [6] a sensor FTC was proposed. In this case the state and faults were estimated using a descriptor observer. In [7] and [8] an observer-based FTC system considering sensor faults was designed. Two observers were used, each one driven by a single sensor to generate residual signals. After isolating the faulty sensor, a switching element was used to select the observer with the healthy sensor. On the other hand, in [9] an actuator $F T C$ for $4 \mathrm{~W}$-steering vehicles was proposed considering a $B M$ with unknown and time-varying cornering stiffness.

These studies show the benefits of using more accurate models, although there are still few results considering the full body dynamics. On the other hand, more complex models are more difficult to manage; therefore, increasing model complexity brings returns only up to a certain point. Nonetheless, there may be operating conditions in which more complex models are indeed required for accurately representing the vehicle behavior. This must be elucidated before the controller design phase. In this regard, the traditional $B M$ has been shown to be adequate for many applications; however, it lacks significant cross-coupling information among the lateral and longitudinal dynamics. In this article, a comprehensive non-linear model is presented and then a linear approximation is derived. The resulting model is shown to be better than the $B M$ for analyzing the lateral and longitudinal cross-coupling while at the same time being relatively simple.

When several VCS operate simultaneously, the performance could also be degraded because of the cross-coupling dynamics. One possibility to deal with these interactions is to take into account the changes in the operating condition of the other subsystems. In [10] a fault-tolerant Linear Time Varying $(L T V)$ controller was designed by considering changes in the longitudinal velocity. In particular, a degraded-mode lateral control for an automated highway system was designed using feedback linearization and a mismatched 
observer synthesized with $\mathcal{H}_{\infty}$. Alternatively, in [11, 12] a Linear Parameter Varying $(L P V) B M$ which considers the velocity as a varying parameter was used for the design of a steering controller.

Another, more direct, alternative to deal with interactions among vehicle subsystems is the synthesis of a centralized controller for several subsystems, an approach called Global Chasis Control [13, 14, 15]. Nevertheless, this is not a trivial task since it involves the use of more complex models and theoretical tools. In addition, centralized controllers usually also require more complex hardware and software for their implementation, and the increased complexity renders them more susceptible to faults. Moreover, typical centralized control schemes do not allow the implementation of physically independent control systems and failure of one subsystem may easily propagate to other subsystems. Due to these difficulties, it is not surprising that the decentralized approach is still predominant in many practical applications $[16,17]$. In this context it would be attractive to retain the advantages of decentralized schemes while at the same time being able to reduce or eliminate the cross-coupling effects.

The literature review reveals that most of the reported $V C S$ deal with decentralized schemes and more recently with schemes that adapt to other subsystem operating conditions. At the same time, reports dealing with centralized control of more than one vehicle subsystem are less widespread at this time, but are increasingly gaining attention. Notwithstanding the current knowledge in the subject, an issue which (up to the best knowledge of the authors) has been practically neglected is the fact that a failure can propagate to other subsystems through cross-coupling.

For instance, when the steering and velocity control systems operate simultaneously and the VelCS fails, several questions may be of interest: Will this failure degrade the performance of the steering subsystem? Can something be done within the steering subsystem to accommodate this failure? Can a FTC steering system be operated independently from the velocity subsystem? It will be shown in this article that a failure of the VelCS indeed propagates to the steering subsystem. However, it is possible to improve the steering response when the VelCS fails. Moreover, this can be achieved without communication between subsystems; i.e. the steering subsystem can accommodate the fault without requiring any additional signal from the velocity subsystem.

Dealing with multiple $V C S$, and decoupling their failures can be very involved because of the required model complexity and vastness of conceivable 
interactions and operating conditions. Although the results presented in this article do not solve all these issues, they do represent an initial approach to the issue through a relevant case study.

The main contributions of this article are: 1) the design of decentralized controllers for the steering and velocity subsystems which are not affected by cross-coupling and 2) a FTC scheme for the steering subsystem which is able to accommodate a failure of the VelCS.

The outline of this paper is as follows: in section 2 , a full vehicle model is presented. Section 3 presents the problem statement, analyzing the limitations of the $B M$ to present the longitudinal-lateral dynamics cross-coupling. In section 4 the cross-coupling is analyzed and nominal controllers for the velocity and steering are synthesized. Section 5 presents the design of a $F D$ scheme for faults in the $\mathrm{VelCS}$ while section 6 presents the $F T C$ scheme for the steering system. The validation of the overall FTC is discussed in section 7 and finally, concluding remarks are presented in section 8. The acronyms and variables used in this article are defined in Table 1 and 2, respectively.

Table 1: Acronyms.

\begin{tabular}{cl}
\hline Acronym & Definition \\
\hline$A B S$ & Anti-lock Braking System \\
$B M$ & Bicycle Model \\
$C C$ & Cruise Control \\
$D L C$ & Double Lane Change \\
$E S C$ & Electronic Stability Control \\
$F D$ & Fault Detection \\
$F N V M$ & Full Nonlinear Vehicle Model \\
$F T C$ & Fault Tolerant Control \\
GCC & Global Chassis Control \\
HbVC & High bandwidth Velocity Controller \\
ICAD & Individual Channel Analysis and Design \\
LbVC & Low bandwidth Velocity Controller \\
LPV & Linear Parameter Varying \\
LTV & Linear Time Varying \\
OL & Open loop \\
$R O C$ & Receiver Operating Characteristic \\
$R L S$ & Recursive Least Squares \\
StC & Steering controller \\
$T-S$ & Takagi-Sugeno \\
VCS & Vehicle Control Systems \\
VelCS & Velocity Control System \\
\hline
\end{tabular}


Table 2: Definition of Variables.

\begin{tabular}{ccc}
\hline Variable & Description & Units \\
\hline$\alpha_{i}, \beta$ & Wheel/vehicle side-slip angle & $\mathrm{deg}$ \\
$\omega_{i}$ & Rotational velocity of the tire $i$ & $\mathrm{rad} / \mathrm{s}$ \\
$\phi, \theta, \psi$ & Roll/pitch/yaw angle & $\mathrm{deg}$ \\
$p, q, r$ & Roll/pitch/yaw rate & $\mathrm{deg} / \mathrm{s}$ \\
$\lambda_{i}$ & Longitudinal slip ratio & - \\
$\delta_{i}$ & Steering wheel angle & $\mathrm{deg}$ \\
$m, m_{s}, m_{u s_{i}}$ & Vehicle/sprung/unsprung mass & $\mathrm{Kg}$ \\
$x, \dot{x}, \ddot{x}$ & Longitudinal displacement, velocity, acceleration & $\mathrm{m}, \mathrm{m} / \mathrm{s}, \mathrm{m} / \mathrm{s}^{2}$ \\
$y, \dot{y}, \ddot{y}$ & Lateral displacement, velocity, acceleration & $\mathrm{m}, \mathrm{m} / \mathrm{s}, \mathrm{m} / \mathrm{s}^{2}$ \\
$z_{u s_{i}}, \dot{z}_{u s_{i}}, \ddot{z}_{u s_{i}}$ & Unsprung mass displacement, velocity, acceleration & $\mathrm{m}, \mathrm{m} / \mathrm{s}, \mathrm{m} / \mathrm{s}^{2}$ \\
$T_{\omega_{i}}$ & Braking torque at the wheel & $\mathrm{N} / \mathrm{m}$ \\
$J_{i}$ & Wheel inertia & $\mathrm{Kgm}$ \\
$I_{x}, I_{y}, I_{z}$ & Roll/pitch/yaw moment of inertia & $\mathrm{Kgm}$ \\
$L$ & Wheelbase & $\mathrm{m}$ \\
$h$ & Height of the Co $\mathrm{m}$ & $\mathrm{m}$ \\
$l_{f}, l_{r}$ & Distance from CoG-front/rear axle & $\mathrm{m}$ \\
$t_{f}, t_{r}$ & Front/rear axle length & $\mathrm{m}$ \\
$k_{s_{i}}, k_{t_{i}}$ & Suspension/tire stiffness coefficient & $\mathrm{N} / \mathrm{m}$ \\
$c_{s_{i}}$ & Damper coefficient & $\mathrm{Ns} / \mathrm{m}$ \\
$R_{i}$ & Effective tire radius & $\mathrm{m}$ \\
\hline
\end{tabular}

\section{Vehicle Model}

\subsection{Full vehicle model}

Vehicles have many subsystems which can interact with each other in certain operating conditions. These interactions can be sufficiently strong to interfere with the effectiveness of decentralized control schemes which only take into consideration the local dynamics. In this context, a vehicle model comprising the relevant dynamic subsystems and their interactions is required for effective $G C C$ analysis and design. Figure 1 presents a model, which comprises the body dynamics (longitudinal, lateral and vertical motions), the suspension system and full tire dynamics (longitudinal, lateral and vertical tire forces). This model allows taking into account several phenomena which have an important influence in the global chassis dynamics; especially in critical driving situations, such as load transfers, nonlinear longitudinal and lateral tire behavior and suspension dynamics. This model will be used to analyze the lateral-longitudinal cross-coupling and to validate the proposed FTC scheme. 


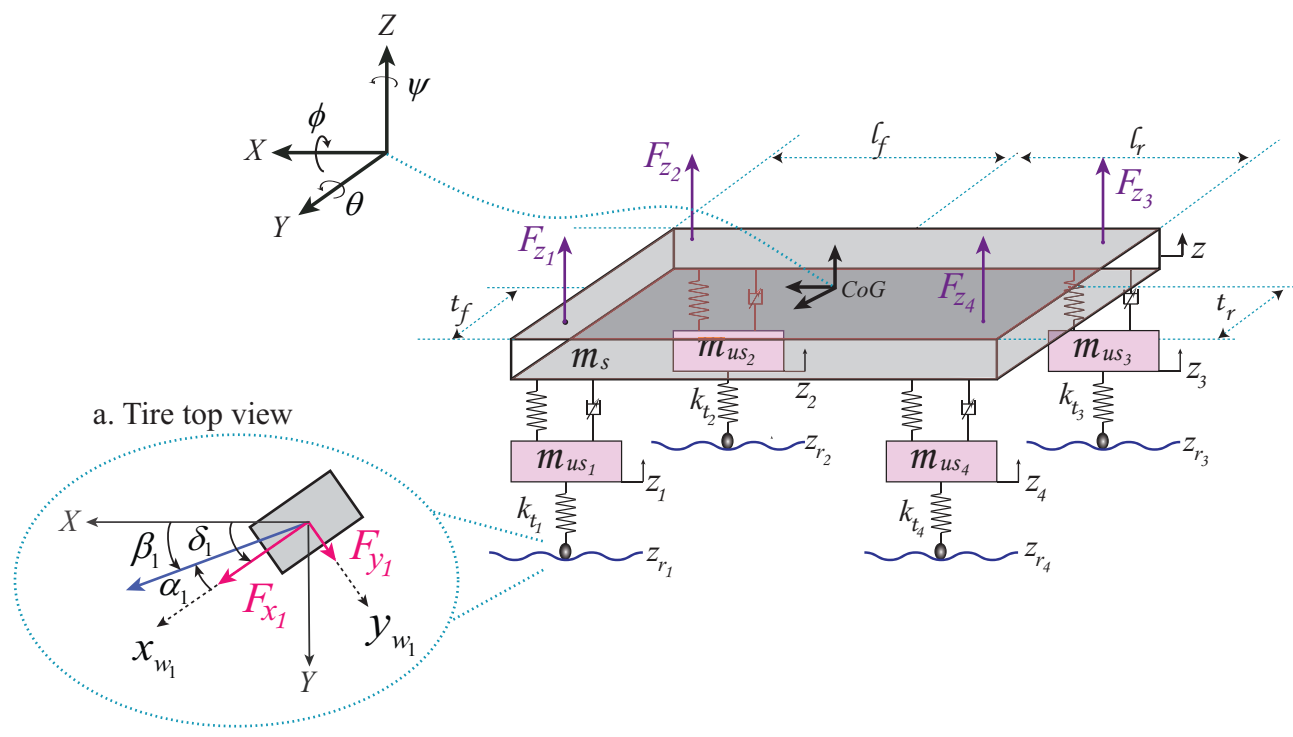

Figure 1: Vehicle reference system

\subsubsection{Dynamical equations}

According to Newton's law, the dynamical equations which model the vehicle motion are:

$$
\begin{aligned}
m \ddot{x}= & F_{x_{1}} \cos \left(\delta_{1}\right)+F_{x_{2}} \cos \left(\delta_{2}\right)+F_{x_{3}}+F_{x_{4}}-F_{y_{1}} \sin \left(\delta_{1}\right) \\
m \ddot{y}= & F_{y_{1}} \sin \cos \left(\delta_{1}\right)+\delta_{y_{2}} \cos \left(\delta_{2}\right)+F_{y_{3}}+F_{y_{4}}+F_{x_{1}} \sin \left(\delta_{1}\right) \\
& +F_{x_{2}} \sin \left(\delta_{2}\right)-m r \dot{x}+m p \dot{z} \\
m \ddot{z}= & F_{z_{1}}+F_{z_{2}}+F_{z_{3}}+F_{z_{4}}-m p \dot{y}+m q \dot{x} \\
I_{x} \dot{p}= & -T_{\omega_{1}} \sin \left(\delta_{1}\right)-T_{\omega_{2}} \sin \left(\delta_{2}\right) \\
& +t_{f}\left(F_{z_{1}}-F_{z_{2}}\right)+t_{f}\left(F_{z_{4}}-F_{z_{3}}\right)+h_{1}\left(F_{y_{1}} \cos \left(\delta_{1}\right)+F_{x_{1}} \sin \left(\delta_{1}\right)\right) \\
& +h_{2}\left(F_{y_{2}} \cos \left(\delta_{2}\right)+F_{x_{2}} \sin \left(\delta_{2}\right)\right)+h_{3} F_{y_{3}}+h_{4} F_{y_{4}}-\left(I_{z}-I_{y}\right) q r \\
I_{y} \dot{q}= & T_{\omega_{1}} \cos \left(\delta_{1}\right)+T_{\omega_{2}} \cos \left(\delta_{2}\right)+T_{\omega_{3}}+T_{\omega_{4}} \\
& -l_{f}\left(F_{z_{1}}+F_{z_{2}}\right)+l_{r}\left(F_{z_{3}}+F_{z_{4}}\right)+h_{1}\left(F_{y_{1}} \sin \left(\delta_{1}\right)-F_{x_{1}} \cos \left(\delta_{1}\right)\right) \\
& +h_{2}\left(F_{y_{2}} \sin \left(\delta_{2}\right)-F_{x_{2}} \cos \left(\delta_{2}\right)\right)-h_{3} F_{x_{3}}-h_{4} F_{x_{4}}-\left(I_{x}-I_{z}\right) p r \\
I_{z} \dot{r}= & l_{f}\left[F_{y_{1}} \cos \left(\delta_{1}\right)+F_{y_{2}} \cos \left(\delta_{2}\right)+F_{x_{1}} \sin \left(\delta_{1}\right)+F_{x_{2}} \sin \left(\delta_{2}\right)\right]-l_{r}\left(F_{y_{3}}+F_{y_{4}}\right) \\
& +t_{f}\left[F_{x_{2}} \cos \left(\delta_{2}\right)+F_{y_{1}} \sin \left(\delta_{1}\right)-F_{x_{1}} \cos \left(\delta_{1}\right)-F_{y_{2}} \sin \left(\delta_{2}\right)\right]+t_{r}\left(F_{x_{3}}-F_{x_{4}}\right) \\
& -\left(I_{y}-I_{x}\right) p q
\end{aligned}
$$

where $F_{x_{i}}$ and $F_{y_{i}}$, with $i=\{1,2,3,4\}$, are the longitudinal and lateral tire forces at each corner respectively, $F_{z_{i}}$ are the suspension forces, $\delta_{i}$ are the 
front wheels steering angles, $T_{\omega_{i}}$ are the breaking/accelerating torques, $h_{i}$ are the heights of the vehicle corners, and $m, m_{s}, t_{f}, t_{r}, r_{i}$ and $I_{i}$ are the physical parameters of the vehicle, Table 2 .

For each wheel, the rotational dynamics and vertical displacement are:

$$
\begin{aligned}
J_{i} \dot{\omega}_{i} & =-F_{x_{i}}+T_{\omega_{i}} \\
m_{u s_{i}} \ddot{z}_{u s_{i}} & =F_{t z_{i}}-F_{z_{i}}
\end{aligned}
$$

where $\omega_{i}$ are the angular velocities of the wheels, $z_{u s_{i}}$ are the vertical displacements of the wheels and $F_{t z_{i}}$ are the tire vertical forces.

\subsubsection{Kinematic relations}

The kinematic equations are due to the vehicle geometry. The vertical positions of the chassis corners $z_{i}$ are:

$$
\begin{array}{ll}
z_{1}=z+t_{f} \sin \phi-l_{f} \sin \theta & z_{2}=z-t_{f} \sin \phi-l_{r} \sin \theta \\
z_{3}=z-t_{r} \sin \phi+l_{r} \sin \theta & z_{4}=z+t_{r} \sin \phi+l_{r} \sin \theta
\end{array}
$$

The slip ratios $\lambda_{i}$ indicate the difference between the angular displacement of each wheel and the translational velocity of the corresponding vehicle corner:

$$
\lambda_{i}=\frac{R_{i} \omega_{i}-V_{t_{i}}}{V_{t_{i}}}
$$

where $V_{t_{i}}=\cos \left(\delta_{i}\right)\left[\dot{x}-q h_{i}-r y_{i}\right]+\sin \left(\delta_{i}\right)\left[\dot{y}+p h_{i}+r x_{i}\right]$

The lateral slip angle at each corner of the vehicle can be calculated as:

$$
\beta_{i}=\tan ^{-1}\left(\frac{\dot{y}+p h_{i}+r x_{i}}{\dot{x}-q h_{i}-r y_{i}}\right)
$$

where $x_{i}=\left\{l_{f}, l_{f},-l_{r},-l_{r}\right\}, y_{i}=\left\{t_{f},-t_{f},-t_{r}, t_{r}\right\}$ and $h_{i}=h+z_{i}-z_{r_{i}}$. The tire side-slip angle is given by $\alpha_{i}=\beta_{i}-\delta_{i}, i=\{1,2,3,4\}$.

Finally, the relation between the front tire angles $\delta_{1}$ and $\delta_{2}$ is given by the Ackerman steering geometry as [18]:

$$
\begin{aligned}
& \delta_{1}=\tan ^{-1}\left(\frac{L}{\left.L \cot (\delta)-t_{f}\right)}\right) \\
& \delta_{2}=\tan ^{-1}\left(\frac{L}{\left.L \cot (\delta)+t_{f}\right)}\right)
\end{aligned}
$$

where $\delta$ is the steering wheel angle. Only front wheel steering is considered, thus $\delta_{3}=\delta_{4}=0$. 


\subsubsection{Tire forces}

The vertical force is given mainly by the tire stiffness:

$$
F_{t z_{i}}=k_{t_{i}}\left(z_{r_{i}}-z_{u s_{i}}\right)
$$

where $z_{r_{i}}$ are the road profile heights at the vehicle corners.

According to the Pacejka model, the forces introduced by the tires at each corner are given by [19]:

- Longitudinal force:

$$
F_{x_{i}}=D_{x} \sin \left[C_{x} \tan ^{-1}\left(B_{x}\left(\left(1-E_{x}\right) \lambda_{i}+\left(E_{x} / B_{x}\right) \tan ^{-1}\left(B_{x} \lambda_{i}\right)\right)\right)\right]
$$

- Lateral force:

$$
F_{y_{i}}=D_{y} \sin \left[C_{y} \tan ^{-1}\left(B_{y}\left(\left(1-E_{y}\right) \alpha_{i}+\left(E_{y} / B_{y}\right) \tan ^{-1}\left(B_{y} \alpha_{i}\right)\right)\right)\right]
$$

where $B_{j}, C_{j}, D_{j}$ and $E_{j}$ are respectively the stiffness, shape, peak and curvature coefficients. These parameters are described as functions of the tire normal force.

\subsubsection{Suspension forces}

The suspension force at each corner is due the spring and the damper:

$$
F_{z_{i}}=k_{s_{i}}\left(z_{u s_{i}}-z_{s_{i}}\right)+c_{s_{i}}\left(z_{u s_{i}}-z_{s_{i}}\right)
$$

\subsubsection{Model Parameters}

A Ford Focus $S T^{T M}$ was used as a case study. Experimental data obtained by a Kinematics $\&$ Compliance $(K \mathscr{G} C)$ test was used to obtain the model parameters, Table 3.

\subsection{Bicycle model}

The $B M$ is a simplification of the main lateral dynamics. A state space representation of the classical $B M$ is given by:

$$
\left[\begin{array}{c}
\dot{\beta} \\
\dot{r}
\end{array}\right]=\left[\begin{array}{ll}
-\frac{c_{r}+c_{f}}{m v} & \frac{c_{r} l_{r}-c_{f} l_{f}}{m v^{2}}-1 \\
\frac{l_{r} c_{r}-l_{f} c_{f}}{I_{z}} & -\frac{l_{f}^{2} c_{f}+l_{r}^{2} c_{r}}{I_{z} v}
\end{array}\right]\left[\begin{array}{l}
\beta \\
r
\end{array}\right]+\left[\begin{array}{c}
\frac{c_{f}}{m v} \\
\frac{l_{f} c_{f}}{I_{z}}
\end{array}\right] \delta
$$

where $c_{f}$ and $c_{r}$ are the cornering stiffness of the front and rear tires, respectively; $v$ is the vehicle longitudinal speed $(\dot{x})$, which is considered constant in this model; and $\beta$ is the vehicle side-slip angle, which is approximated by $\beta \approx \dot{y} / v$. 
Table 3: Vehicle parameters (Ford Focus $S T^{T M}$ ).

\begin{tabular}{cccc}
\hline Parameter & Value & Parameter & Value \\
\hline$m$ & $1507.97 \mathrm{Kg}$ & $m_{s}$ & $1331.98 \mathrm{Kg}$ \\
$m_{u s_{1,2}}$ & $45.495 \mathrm{Kg}$ & $m_{u s_{3,4}}$ & $42.50 \mathrm{Kg}$ \\
$l_{f}$ & $1.05962 \mathrm{~m}$ & $l_{r}$ & $1.58943 \mathrm{~m}$ \\
$t_{f}$ & $0.77565 \mathrm{~m}$ & $t_{r}$ & $0.76386 \mathrm{~m}$ \\
$I_{x}$ & $471.5489 \mathrm{Kgm}^{2}$ & $I_{y}$ & $2757.4032 \mathrm{Kgm}^{2}$ \\
$I_{z}$ & $2997.7942 \mathrm{Kgm}^{2}$ & $J$ & $0.90 \mathrm{Kgm}^{2}$ \\
$k_{s_{1,2}}$ & $37027 \mathrm{~N} / \mathrm{m}$ & $k_{s_{3,4}}$ & $32240 \mathrm{~N} / \mathrm{m}$ \\
$c_{s}$ & $3264 \mathrm{Ns} / \mathrm{m}$ & $k_{t}$ & $318898 \mathrm{~N} / \mathrm{m}$ \\
$R_{i}$ & $0.3062 \mathrm{~m}$ & $h$ & $0.5715 \mathrm{~m}$ \\
\hline
\end{tabular}

\section{Problem Statement}

\subsection{Open-loop Steering Response}

Traditionally, the $B M$ is used to describe the vehicle lateral dynamics; however, it will be shown that this model fails to capture important dynamical interactions among vehicle subsystems.

Consider the following driving conditions: a vehicle running at $100 \mathrm{~km} / \mathrm{h}$ with a steering angle of $2.5^{\circ}$. Fig. 2 shows the yaw rate response of due to a decrement of $0.5^{\circ}$ in the steering angle using several vehicle models. The $B M$ predicts a typical slight under-steered response. However, the Open Loop $(O L)$ response of the Full Nonlinear Vehicle Model (FNVM) is significantly different from that of the $B M$. Figure 2 also shows the response of the linear approximation of the FNVM $\left(O L^{*}\right)$ around the aforementioned operating condition.

The main differences between the responses of the $B M$ and the FNVM $(O L)$ are due to the velocity variation observed in the full model response, Fig. 3, while the $B M$ assumes a constant velocity. In addition, it can be observed that the linear approximation is able to capture this behavior closely.

Under-steer, neutral-steer or over-steer specifications are commonly used to define the desired vehicle handling qualities. In particular, neutral-steer or slightly under-steer specifications (i.e. slight or no overshoot) are common for this type of vehicles. Therefore, it is important for the vehicle to comply with this dynamical behavior. In the following sections the use of decentralized controllers for the steering subsystem will be studied to explore the possibility of achieving a neutral-steer condition. 


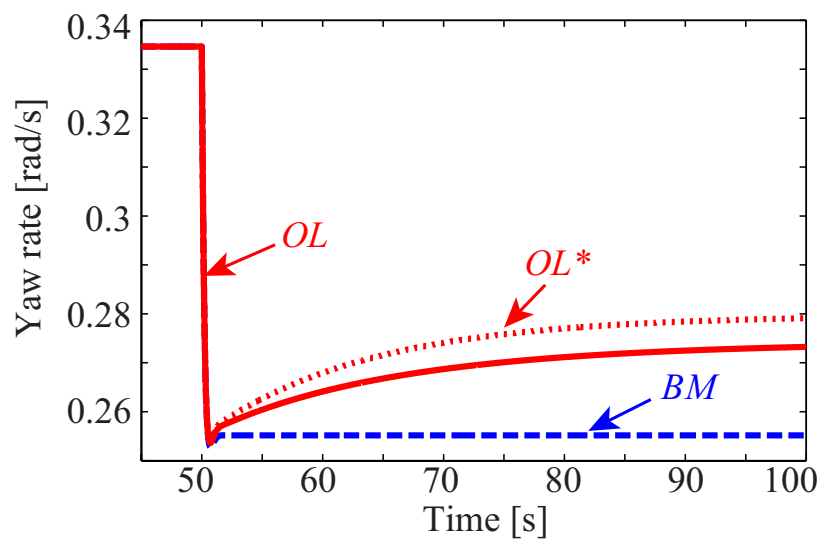

Figure 2: Open loop yaw rate response of the $B M$ and the $F N V M$ to a decrement of $0.5^{\circ}$ in the steering input.

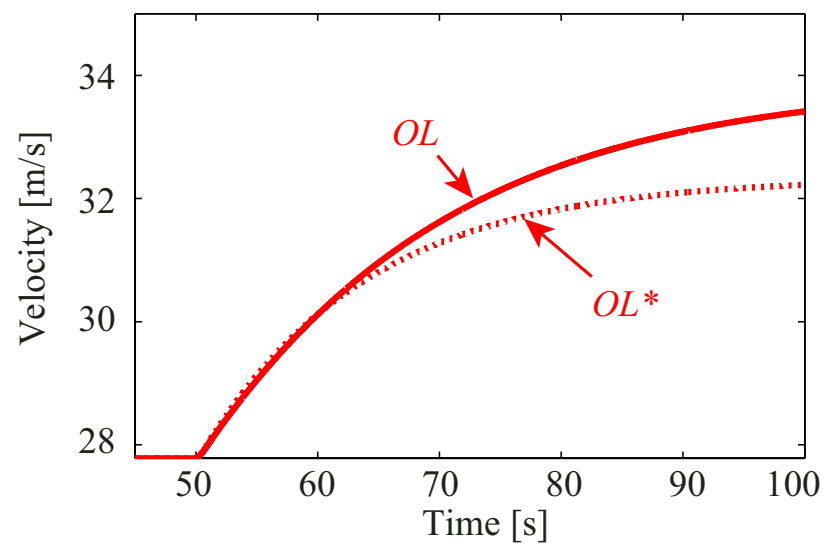

Figure 3: Longitudinal velocity response of the $F N V M$ due to a decrement of $0.5^{\circ}$ in the steering input.

\subsection{Standalone steering control}

A decentralized controller for the yaw rate can be designed to improve the yaw response in a cornering situation. For this type of vehicle the preferred response is that of a neutral-steering setting. Figure 4 shows a comparison of the yaw rate response when using a steering controller $(S t C)$ (designed in section 4.2) and the open loop response (OL), both considering the FNVM.

From this comparison it is clear that the response of the vehicle is improved with respect to the open loop case. In addition, the closed loop response is similar to that of a neutral-steer vehicle, and it is better in this 

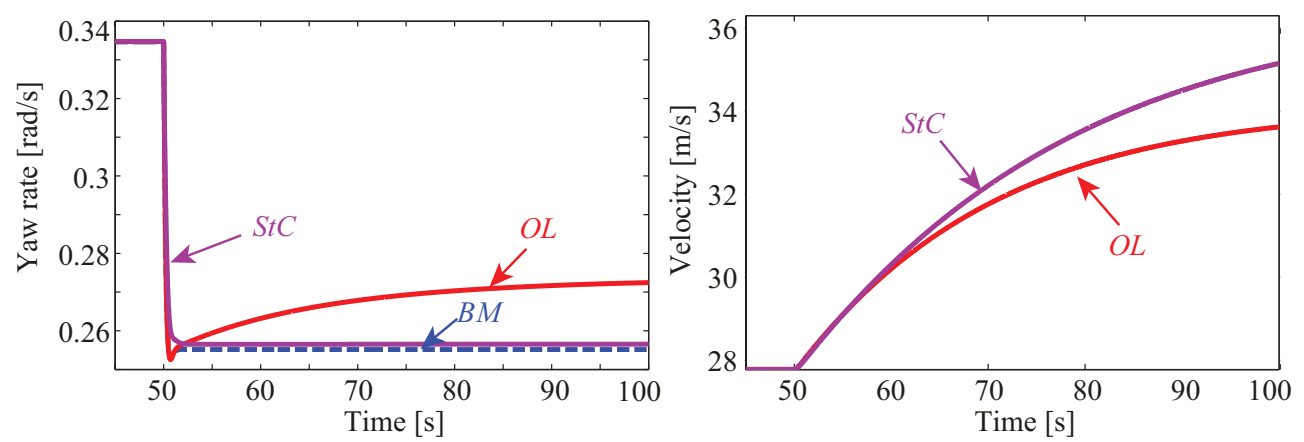

Figure 4: Vehicle response for a decrement of $0.5^{\circ}$ in the steering input when using a steering control $(S t C)$ and in open loop $(O L)$ : (left) yaw rate, (right) longitudinal velocity.

sense that the open loop $B M$ response. Nevertheless, the disadvantage of this approach can be observed in the velocity response of the vehicle, Fig. 4(right). When the yaw rate is operated in closed loop $(S t C)$ the velocity is more affected than in the open loop configuration $(O L)$. Such a variation of velocity in a maneuver is unrealistic because even if the vehicle did not have an automatic velocity control, the driver could make corrective actions trying to maintain an approximately constant velocity. This shows that even if an effective steering control is used, it is still necessary to consider the cross-coupling between the lateral and velocity dynamics.

\subsection{Standalone velocity control}

The $B M$ was derived considering a constant velocity, thus it is reasonable to think that a control scheme capable of maintaining a constant velocity could be used to obtain a behavior similar to that of the $B M$.

First, a high bandwidth VelCS (designed in section 4.1), is considered while the steering system is operated in open loop. Figure 5(left) shows the yaw rate response to a decrement of $0.5^{\circ}$ in the steering angle. Even when using a high bandwidth $(1,200 \mathrm{rad} / \mathrm{s})$ velocity controller $(H b V C)$, the yaw rate is not exactly equal to that $B M$. This difference is due to the effect of the traction moments in the steering which are present even when the velocity is kept constant, i.e. there is a steady state cross-coupling between the yaw rate and the velocity. Nevertheless, the main dynamical characteristics of both responses are similar.

The response of the forward velocity during this maneuver is shown in Fig. 5(right). The high bandwidth VelCS rejects the cross-coupling from 

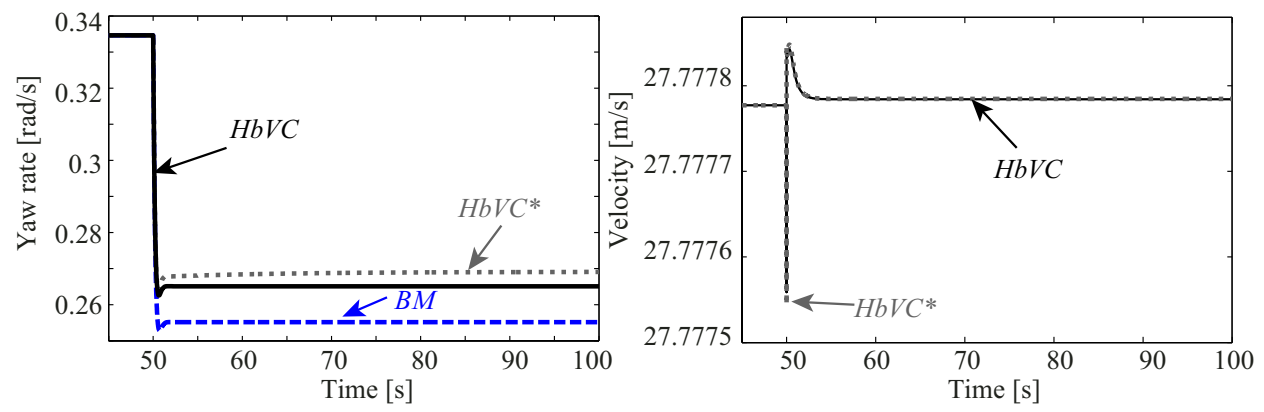

Figure 5: Vehicle response for a decrement of $0.5^{\circ}$ in the steering input when using a high bandwidth velocity control $(H b V C$ ): (left) yaw rate, (right) longitudinal velocity.

the steering maneuver in such a level that the velocity can be considered as constant. The problem with a high bandwidth controller is that its implementation is unfeasible because of actuator bandwidth limitations. In general, the actuator bandwidth should be greater than that of the control system to avoid stability and performance problems due to delay and low damping. In this case the actuator is the powertrain, whose bandwidth is limited by mechanical restraints that yield a maximum bandwidth between 0.808 to $6.8 \mathrm{rad} / \mathrm{s}$, depending on the operating conditions, [20].

Figure 6(right) shows the comparison of the velocity response when a steering maneuver of $0.5^{\circ}$ is performed using a lower bandwidth, more realistic, velocity control $(L b V C)$. The VelCS effectively rejects the effect of the steering maneuver as shown in Fig. 6(right). However, due to the particular cross-coupling characteristics of the vehicle, a lower bandwidth velocity controller has a greater influence over the steering. This results in a severely under-steered yaw rate response in comparison with the open loop $B M$ model, Fig. 6(left).

Figures 5 and 6 also show that the linear approximations (i.e. $H b V C^{*}$ and $L b V C^{*}$ ) adequately capture the main characteristics of the FNVM for both yaw rate and velocity, including the cross-coupling dynamics.

\subsection{Discussion}

It is clear that the $B M$ alone it is not enough to analyze the vehicle behavior in conditions when the velocity is also affected. In addition, a VelCS with realistic bandwidth, implemented either by an automatic velocity control system or by a driver, can have a significant effect in the steering response due to the cross-coupling. 

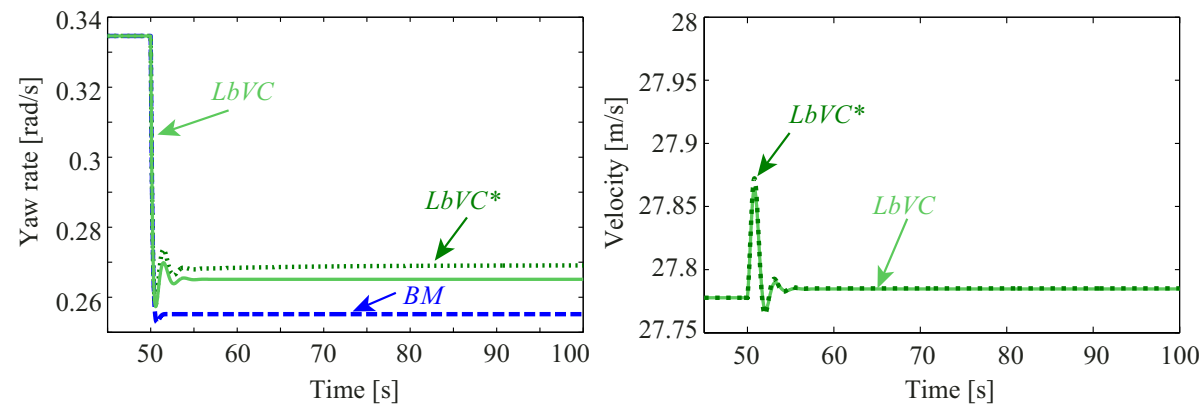

Figure 6: Vehicle response for a steering input of $0.5^{\circ}$ when using a lower bandwidth velocity control $(L b V C)$ : (left) yaw rate, (right) longitudinal velocity.

These results suggest that the best option may be to control both steering and velocity simultaneously. Although a centralized $G C C$ has the advantage of intrinsically considering cross-coupling, distributed controllers are easier to design and implement. Therefore, the steering and velocity controllers will be obtained using a multivariable framework that allows designing decentralized controllers which are less affected by cross-coupling.

Besides affecting the normal operation of the control subsystems, the cross-coupling also plays an important part in faulty scenarios. Therefore, faults in the VelCS that result in an open loop velocity operation will also be considered. That is, events which deactivate the velocity controller. It will be shown that these faults have an adverse effect on the steering response through cross-coupling and require active accommodation. Since decentralized operation is desirable, a fault detection and accommodation scheme for faults in the VelCS which can operate within the steering control subsystem will be presented; that is, without requiring any additional data from the velocity control subsystem. This allows a true decentralized operation of the steering control subsystem.

Finally, the previous simulations show that the linear approximation of the FNVM is much more accurate than the $B M$, particularly for the crosscoupling. Since the FNVM is rather complex for practical analysis, then the linear approximation will be used for the theoretical analysis and controller design. Nonetheless, in all cases the resulting control schemes are validated later using the FNVM. 


\section{Cross-coupling analysis and control design}

In [21] it is shown that a decentralized two-inputs two-outputs $(2 \times 2)$ control system, such as the one presented in Fig.7(left), can be decomposed as shown in Fig.7(right) where:

$$
\begin{aligned}
\Gamma_{H 1}(s) \triangleq \frac{g_{12}(s) g_{21}(s)}{g_{11}(s)}\left(\frac{k_{2}(s)}{1+k_{2}(s) g_{22}(s)}\right) \\
\Gamma_{H 2}(s) \triangleq \frac{g_{12}(s) g_{21}(s)}{g_{22}(s)}\left(\frac{k_{1}(s)}{1+k_{1}(s) g_{11}(s)}\right)
\end{aligned}
$$
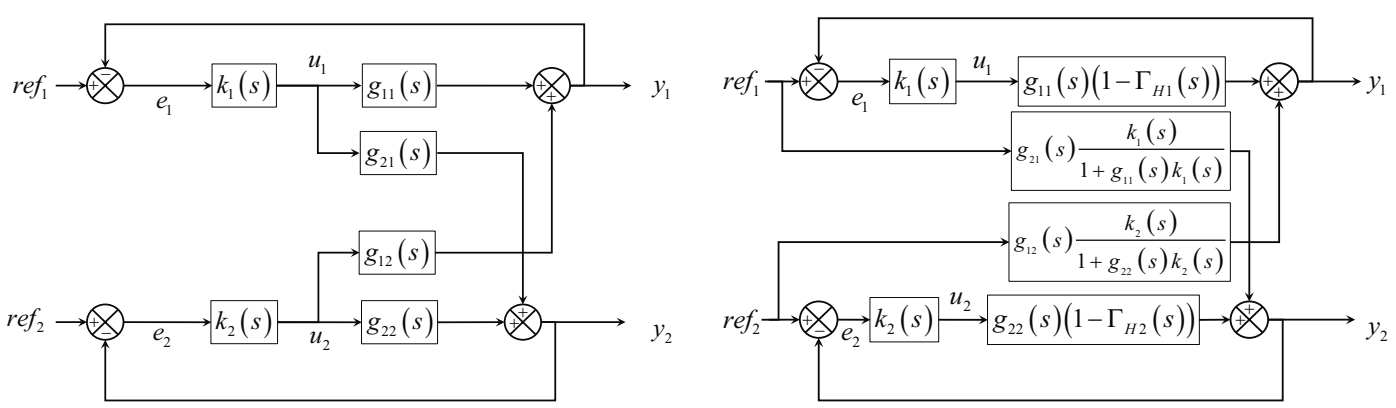

Figure 7: Representation of a 2x2 MIMO system

It is possible to analyze the velocity and steering control problem in the previous structure by considering:

- $u_{1}=T_{\omega}=T_{\omega_{1}}+T_{\omega_{2}}$ : accelerating moment of the front wheels

- $u_{2}=\delta$ : steering wheel angle

- $y_{1}=v=\dot{x}$ : longitudinal velocity (body frame)

- $y_{2}=r$ : yaw rate

- $k_{1}(s)=k_{\delta}(s)$ : steering controller

- $k_{2}(s)=k_{v}(s)$ : velocity controller

Accordingly, the linear approximation of the full vehicle dynamics considering the input vector $U=\left[\begin{array}{ll}u_{1} & u_{2}\end{array}\right]^{\top}$ and the output vector $Y=\left[\begin{array}{ll}y_{1} & y_{2}\end{array}\right]^{\top}$ can be written as:

$$
\left[\begin{array}{l}
v(s) \\
r(s)
\end{array}\right]=\left[\begin{array}{ll}
g_{v, T_{\omega}}(s) & g_{v, \delta}(s) \\
g_{r, T_{\omega}}(s) & g_{r, \delta}(s)
\end{array}\right]\left[\begin{array}{c}
T_{\omega}(s) \\
\delta(s)
\end{array}\right]
$$


In order to analyze the effect of the velocity controller over the yaw rate, the individual channel equation (in accordance with Fig.7) can be used:

$$
\frac{r(s)}{\delta(s)}=g_{r \delta}(s)\left(1-\Gamma_{H 1}(s)\right) \triangleq C_{r}(s)
$$

where:

$$
\Gamma_{H 1}(s) \triangleq \frac{g_{r T_{w}}(s) g_{v \delta}(s)}{g_{r \delta}(s)}\left(\frac{k_{v}(s)}{1+k_{v}(s) g_{v T_{w}}(s)}\right)
$$

Note that if $k_{v}(s)=0$ (i.e. the velocity is operated in open loop) then $C_{r}(s)=g_{r \delta}(s)$ as expected. On the other hand, if the velocity is operated in closed loop, the term $\Gamma_{H 1}(s)$ measures how the steering open loop response is modified through cross-coupling. Equation (18) is known as the Multivariable Structure Function within the ICAD theory [21]. It is known that a high magnitude in $\Gamma_{H 1}\left(j \omega_{0}\right)$ indicates a high cross-coupling at frequency $\omega_{0}$.

It is clear that (18) depends on the velocity controller. In the following sections, the effect of the velocity controller characteristics over the crosscoupling will be assessed with the aid of this equation.

\subsection{Velocity controller design}

A $H b V C$ was designed using $g_{v, T_{\omega}}(s)$ as a design model and classical frequency analysis tools. The specifications of this controller are: a) bandwidth greater than $1000 \mathrm{rad} / \mathrm{s}, \mathrm{b}$ ) steady state tracking error lower than $0.01 \%$ and c) fast rise time. The resulting controller is given by:

$$
k_{v_{H b}}(s)=-\frac{2.985 \times 10^{5}\left(s^{2}+11.5 s+15\right)}{s^{2}+5.1 s+0.5}
$$

Note that the sign of eqn. (19) is due to the fact that a negative $T_{\omega}$ implies an accelerating traction in this model.

Figure 8(left) shows the open loop frequency response of the yaw rate in two conditions: 1) when the velocity is operated in open loop 2) when the velocity is operated in closed loop using the $H b V C$. This figure shows that the main differences occur at low frequencies. Accordingly, the step response of the two systems (Fig.8(right)) confirms this assessment.

Now a velocity controller with realistic bandwidth and performance is considered $(L b V C)$. This controller is designed with the following specifications [22]: a) bandwidth between 1.5-3 rad/s, b) steady state tracking error lower than $0.01 \%$ and c) fast rise time. The resulting controller is given by: 
Frequency response

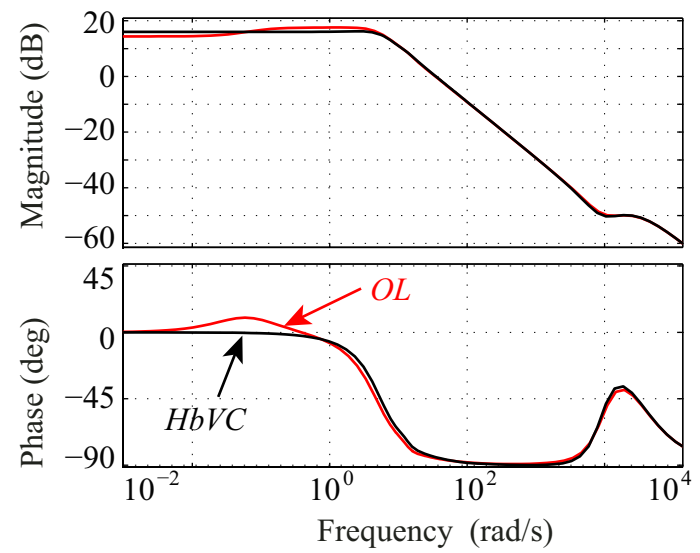

Time response

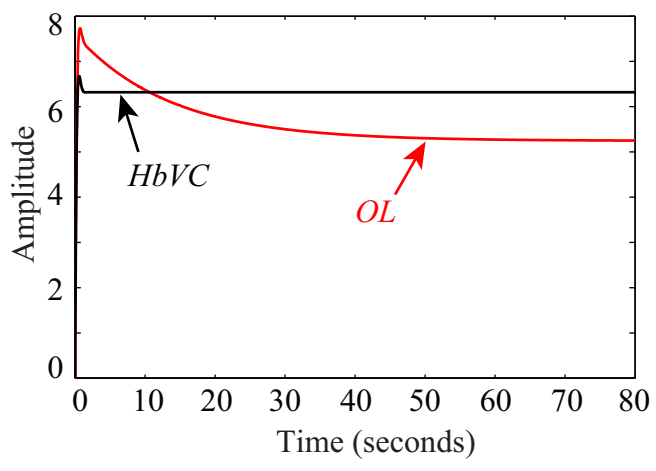

Figure 8: Open loop yaw rate response when the velocity is operated in open loop $(O L)$ and with a high-bandwidth controller $(H b V C)$.

$$
k_{v_{L b}}(s)=-\frac{2985 s+4478}{s^{2}+5.1 s+0.5}
$$

Figure 9 shows the comparison of the magnitude of $\Gamma_{H 1}(s)$ when considering the high and low bandwidth velocity controllers. First, when using the $H b V C$ the cross-coupling affects mainly the low frequencies. This is in line with the observations made from Fig. 8. On the other hand, the $L b V C$ lowers the coupling for higher frequencies and increases the cross-coupling around the velocity controller bandwidth. This is to be expected because it is widely known that the highest sensitivity of a control system is around its bandwidth.

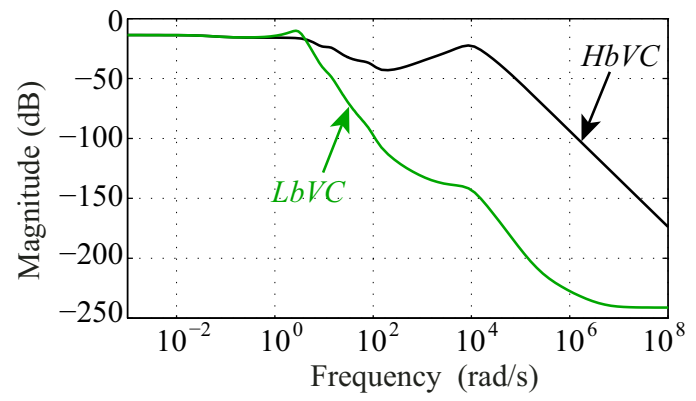

Figure 9: Magnitude of $\Gamma_{H 1}(s)$ when considering the high and low bandwidth velocity controllers. 
The increased cross-coupling of the $L b V C$ affects the yaw-rate response further. Figure 10(left) presents a comparison of the open-loop frequency response of the yaw rate when the velocity is operated in: 1) open loop, 2) in closed loop using the $H b V C$ and 3) in closed loop using the $L b V C$. The effect of the $L b V C$ is the addition of a medium frequency resonance in the yaw rate response, while at low frequencies it remains very similar as when using the $H b V C$. The effect in the time domain can be readily seen in the step responses of Fig. 10(right).

Frequency response

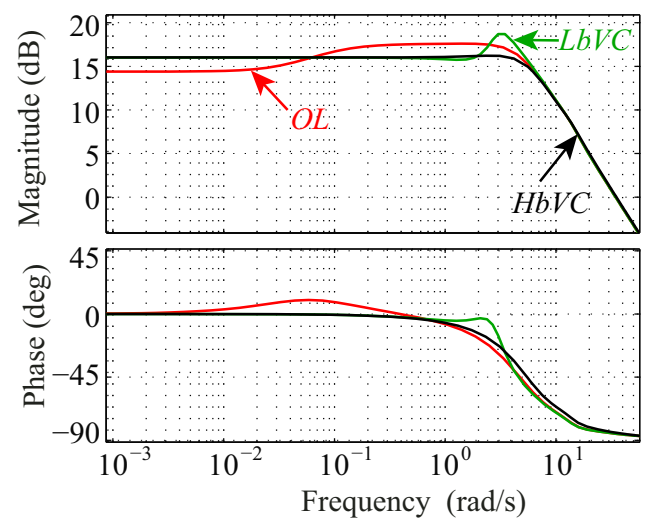

Step response

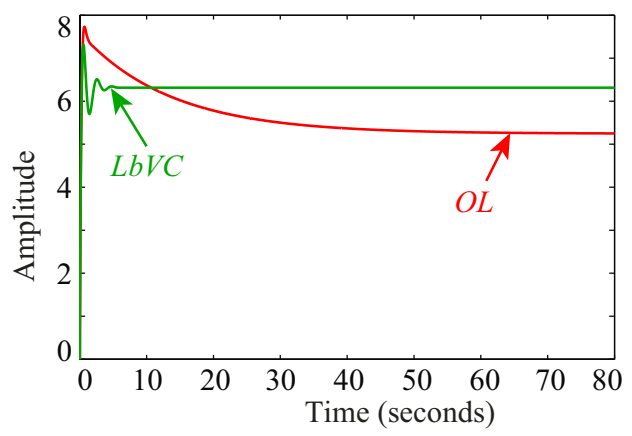

Figure 10: Open loop yaw rate response when the velocity is operated in open loop $(O L)$ and with the low-bandwidth controller $(L b V C)$.

The analysis shows that the open loop yaw rate dynamics are clearly affected by the cross-coupling when using a velocity controller of realistic bandwidth. This will be addressed by designing a proper steering controller in the next section.

\subsection{Steering controller design}

A steering controller is designed to improve the vehicle safety properties to avoid loss of maneuverability; in particular, a neutral-steer response is desired. This can be translated to: a) almost critically damped response (around $70^{\circ}-90^{\circ}$ of phase margin) b) bandwidth around 2-4 rad/s, c) steady state tracking error $e(t)=\left(r_{d}(t)-r(t)\right) / r_{d}(t)<0.1$, [23].

It would be also possible to design a controller with a higher bandwidth that follows a reference model, as in [24]; this is akin to using a reference pre- 
filter. However, this requires higher bandwidth actuators and sensors which may be unavailable. Moreover, the actual response to perturbations would follow the internal high bandwidth response instead of the desired overall reference dynamic, [25]. Therefore, it is proposed to achieve the required neutral-steer response directly by the closed loop dynamics, reducing the need for pre-filtering elements or reference model. The yaw rate reference signal is set to [26]:

$$
r_{d}=\frac{v}{L} \delta_{\text {driver }}
$$

where $\delta_{\text {driver }}$ is the driver steering angle command.

Additional requirements for easy implementation of the controller are: d) stable and minimum phase controller, e) low order and f) without high frequency modes (i.e. much higher that the control loop bandwidth).

As noted before, the main problem when designing the steering controller is that a low bandwidth velocity controller introduces a high-level of crosscoupling around the effective frequency range. At first, consider the Steering Controller 1 (StC1):

$$
k_{\delta_{1}}(s)=\frac{0.1935 s^{3}+21.86 s^{2}+425.7 s+2264}{s^{3}+145.1 s^{2}+4510 s+315}
$$

which was designed using classical frequency analysis tools and considering $C_{r}(s)=g_{r, d}(s)$; that is, assuming that the velocity is operated in open loop. Figure 11 shows the open loop Bode diagram and the closed loop step responses of the yaw rate using controller StC1 in two different scenarios. In the first case the velocity is operated in open loop $(O L V)$; that is, only steering is controlled. In this configuration controller StrC1 is able to achieve the requirements for the yaw rate. In the second case the velocity is operated in closed loop using the $L b V C$; that is, both steering and velocity are controlled in a decentralized manner. In this scenario the increased cross-coupling from the velocity controller introduces a slight resonance, which is not completely eliminated by the steering control loop.

The Double Lane Change ( $D L C)$ maneuver is commonly used to assess the handling qualities of the lateral dynamics. Therefore, in order to validate the previous observations, both cases are simulated using the full nonlinear model with a $(D L C)$ test. Figure 12 shows that the yaw rate response of the FNVM has the same characteristics observed in the linearized model. When the velocity is operated in open loop, the steering controller (22) is 
Frequency response

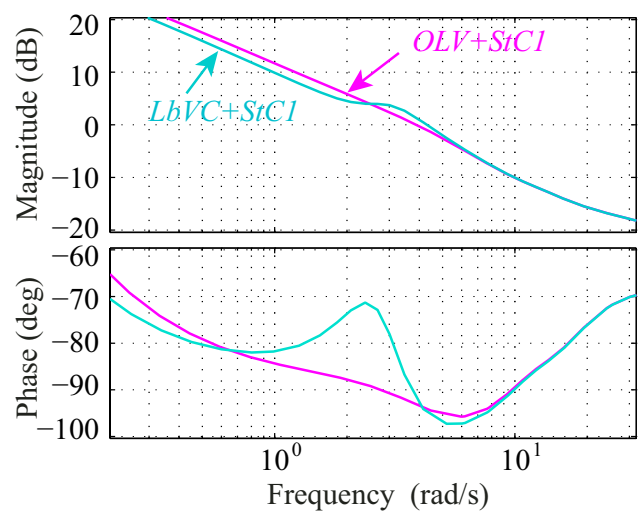

Step response

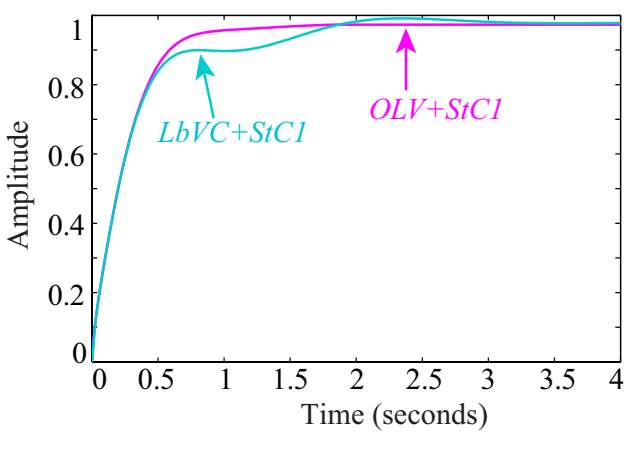

Figure 11: Comparison of the yaw rate response with the steering controller of eqn. (22) when the velocity is operated: in open loop $(O L V+S t C 1)$ and with the low-bandwidth velocity controller $(L b V C+S t C 1)$.

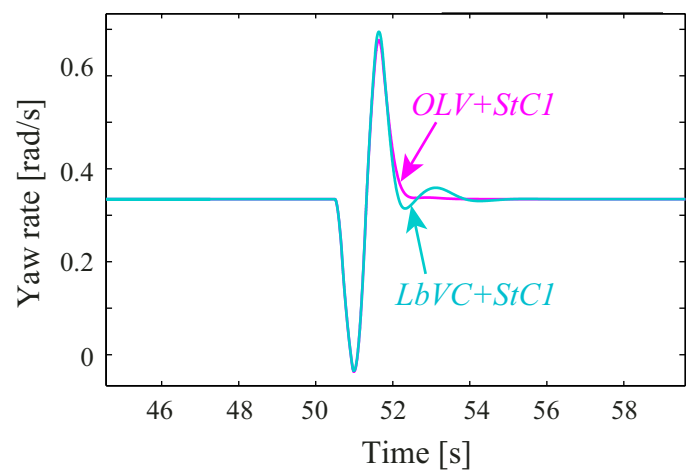

Figure 12: Yaw rate response in the $D L C$ test with the steering controller of eqn. (22) when the velocity is operated: in open loop $(O L V+S t C 1)$ and with the low-bandwidth velocity controller $(L b V C+S t C 1)$.

able to achieve the requirements for the yaw rate; whereas when the velocity is operated in closed loop, an unwanted oscillation can be observed.

Since the performance of the steering controller is deteriorated when the vehicle operated with the velocity in closed loop, a different controller is designed for the steering control system considering that the velocity is operated in closed loop with the $L b V C$. To take into account the cross-coupling, the yaw rate controller is designed using: 


$$
C_{r}(s)=g_{r \delta}(s)\left(1-\frac{g_{r T_{w}}(s) g_{v \delta}(s)}{g_{r \delta}(s)} \frac{k_{v}(s)}{1+k_{v}(s) g_{v T_{w}}(s)}\right)
$$

and considering the same specifications. The resulting Steering Controller 2 (StC2) is given by:

$$
k_{\delta_{2}}(s)=\frac{0.05 s^{3}+0.5486 s^{2}+1.343 s+4.106}{s^{3}+2.447 s^{2}+7.302 s+0.7067}
$$

Figure 13 shows the open loop Bode diagram and the closed loop step responses of the yaw rate using controller StC2 in two conditions, when the velocity is operated in open loop $(O L V)$ and in closed loop with the $L b V C$. In particular, Fig. 13a shows that controller StC2 compensates the effect of cross-coupling when the $L b V C$ is used. However, when the velocity is operated in open loop, the opposite condition is observed. This controller is only appropriate when the velocity system is operating in closed loop.
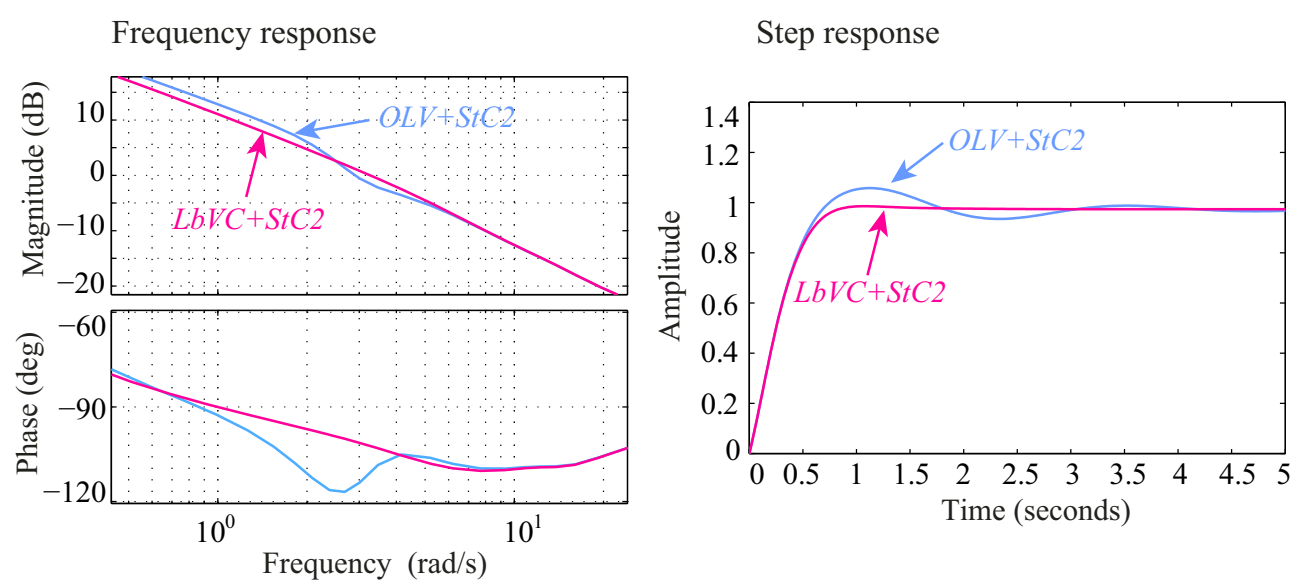

Figure 13: Comparison of the yaw rate response with the steering controller eqn. (24) when the velocity is operated: in open loop $(O L V+S t C 2)$ and with the low-bandwidth velocity controller $(L b V C+S t C 2)$.

This controller is also assessed with the $D L C$ test and the FNVM. Figure 14 shows the yaw rate response when the vehicle velocity is operated in open and closed loop. When the velocity system is operated in closed loop and the StC2 controller is used, the yaw rate complies with the requirements. However, if the velocity control loop is deactivated, an oscillation is induced 


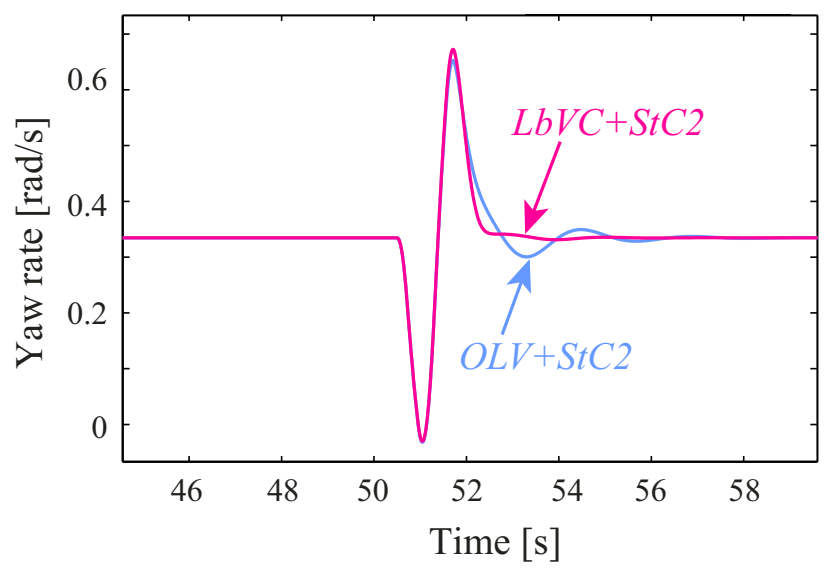

Figure 14: Yaw rate response in the $D L C$ test with the steering controller of eqn. (24) when the velocity is operated: in open loop $(O L V+S t C 2)$ and with the low-bandwidth velocity controller $(\mathrm{Lb} V \mathrm{C}+\mathrm{StC} 2)$.

in the yaw rate response. This confirms the behavior observed using the linearized model.

Considering the discussion of Figs. 12 and 14, depending on the condition in which the $\mathrm{VelCS}$ is operated, the appropriate yaw rate controller must be used to achieve the desired performance. The cross-coupling between the velocity and yaw-rate control systems is such that the same steering controller cannot be used when the velocity is operated in open and in closed loop. Thus, two different steering controllers are used according to the functionality of the VelCS as follows:

- Case 1: StC1 when the velocity is operated in open loop.

- Case 2: StC2 when the velocity is operated in closed loop.

These controllers are required only for achieving the highest performance; nevertheless, in all cases the stability is warranted.

\section{Detection of faults in the longitudinal dynamics}

To apply the appropriate controller for the lateral dynamics, a Fault Detection $(F D)$ module is designed to determine if the vehicle is operating with or without velocity control. The proposed novel approach is that the functionality of the $\mathrm{VelCS}$ is diagnosed by monitoring exclusively the behavior of the vehicle lateral dynamics. 
When the VelCS is deactivated because of faults in sensors or actuators, the yaw rate dynamics tend to present a slow mode. Considering this observation, a $F D$ module based on parametric estimation is proposed. The inputs of the detection module are the steering angle and the yaw rate. The Recursive Least Squares $(R L S)$ algorithm with forgetting factor is used to estimate a second order discrete-time approximation of transfer function $C_{r}(s)$. Figure 15 shows the real part of the estimated poles when faults are induced at $t \in\{[0,800],[1600,2400]\}$. It is clear that when a fault in the VelCS occurs, the parametric estimator identifies slower poles than in the nominal condition.

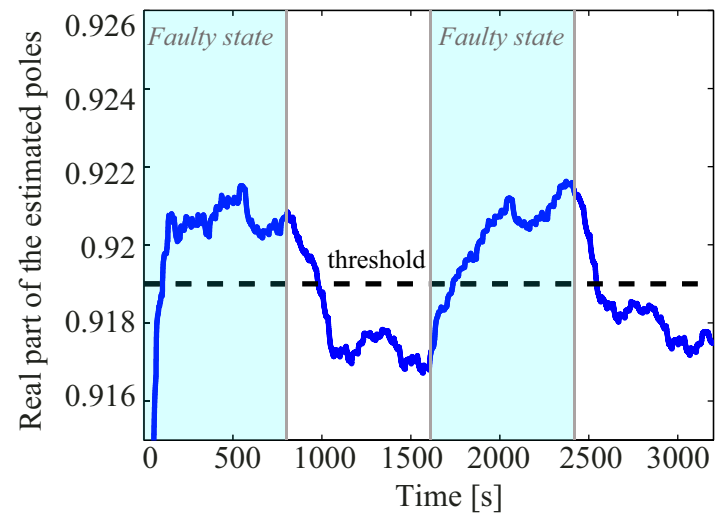

Figure 15: Estimation of the real part of the discrete-time poles to detect faults in the velocity control loop.

By defining a threshold, fault detection of the velocity control system is achieved. The output of the $F D$ module is $F D=0$ for the healthy case (i.e when the velocity operates in closed loop) and, conversely, $F D=1$ when a fault in the VelCS occurs (i.e the velocity is in open loop). Figure 16 show the Receiver Operating Characteristic (ROC) curve of the FD module for different threshold values. In this figure, the closer the curve to the point $(0,1)$, the better the classification is. As observed, the threshold at $t h=0.9186$ presents the best detection performance.

Figure 17 shows the results of the FD module. The detection error, which is computed as the percentage of miss-estimation time relative to the total time, is $17.7 \%$. 


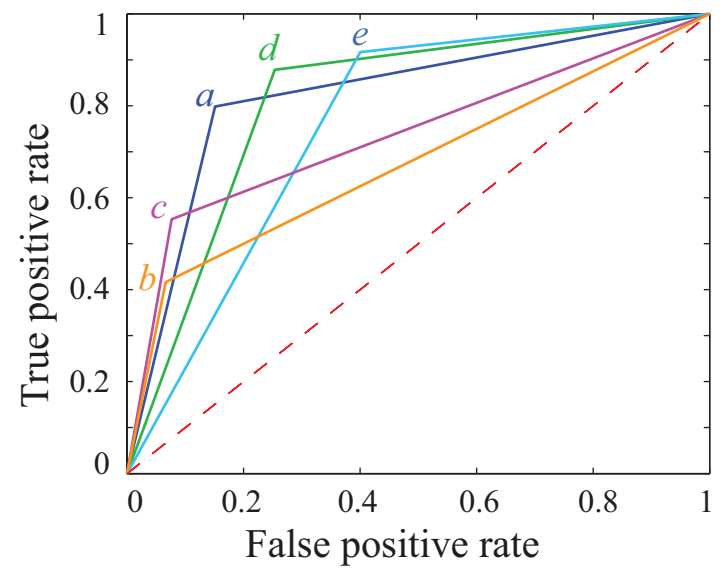

Figure 16: $R O C$ curve of the $F D$ module for different thresholds: a) $t h=0.9186$, b) $0.85 t h$, c) $0.9 t h, d) 1.1 t h$, e) $1.15 t h$.

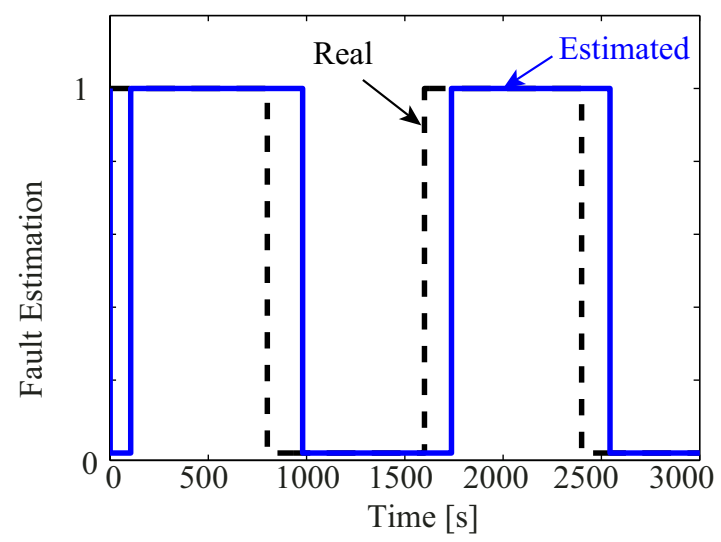

Figure 17: Detection of faults in the velocity control loop based on parameter estimation.

\section{FTC Strategy}

By using the information of the $F D$ module, the steering controller is scheduled in accordance with the VelCS functionality. The resulting steering controller is given by:

$$
k_{\delta}(s)=\left\{\begin{array}{lll}
\frac{0.05 s^{3}+0.5486 s^{2}+1.343 s+4.106}{s^{3}+2.447 s^{2}+7.302 s+0.7067} & \text { if } & F D=0 \\
\frac{0.19355^{3}+21.86 s^{2}+425.7 s+2264}{s^{3}+145.1 s^{2}+4510 s+315} & \text { if } & F D=1
\end{array}\right.
$$

Figure 18 shows the block diagram of the FTC scheme. This architecture 


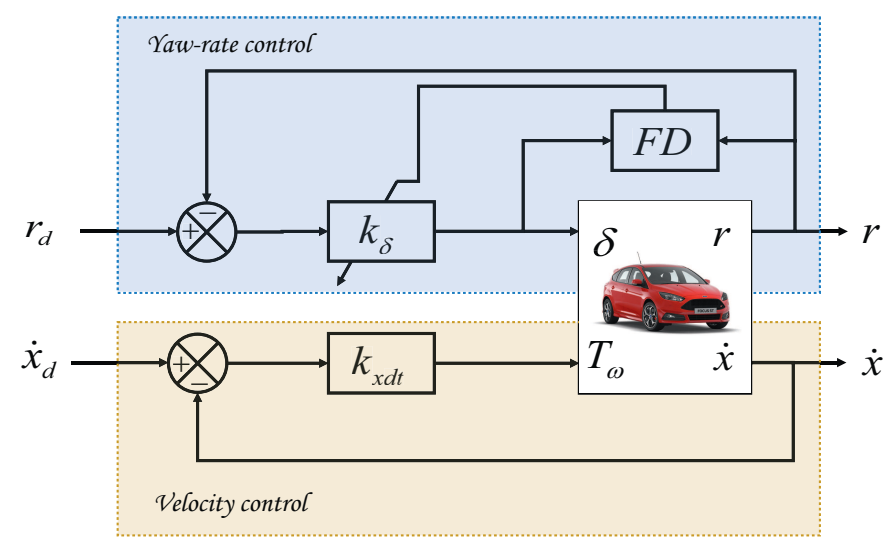

Figure 18: Block diagram of the FTC strategy.

includes the velocity and steering controllers as well as the $F D$ module. Note that the yaw rate and velocity controllers, including the $F D$ module are fully independent.

\section{FTC strategy assessement}

In this section the complete control strategy is tested via simulation using the FNVM. The key characteristics that must be assessed to ensure the reliability of the proposed strategy are:

1. Functionally of the $F D$ module.

2. Stability of the scheduling scheme.

3. Stability of lateral response when the $F D$ module operates in any detection (either false or true) condition.

4. Improved lateral performance when the $F D$ module operates in either true-positive or true-negative condition.

5. Adequate velocity regulation when the $\operatorname{VelCS}$ is healthy.

In order to evaluate these elements, the following test is devised. The test starts with the vehicle driving at $100 \mathrm{~km} / \mathrm{h}$ with an steering angle of $2.5^{\circ}$. The velocity system is operated in closed loop, then a fault which deactivates the VelCS is simulated at time $t=900 \mathrm{~s}$. To evaluate the performance of the yaw-rate $F T C$, two $D L C$ maneuvers are performed at times $t=705 \mathrm{~s}$ and $t=1605 \mathrm{~s}$. The $F D$ module requires excitation of the lateral dynamics to detect the fault. In the simulation this is achieved by establishing a 
low amplitude pseudo-random sequence for the yaw rate reference. This reference is maintained at all times except when the two $D L C$ maneuvers are performed. In addition, the $F D$ module is initialized with a false positive state. The resulting responses are summarized in Fig. 19.

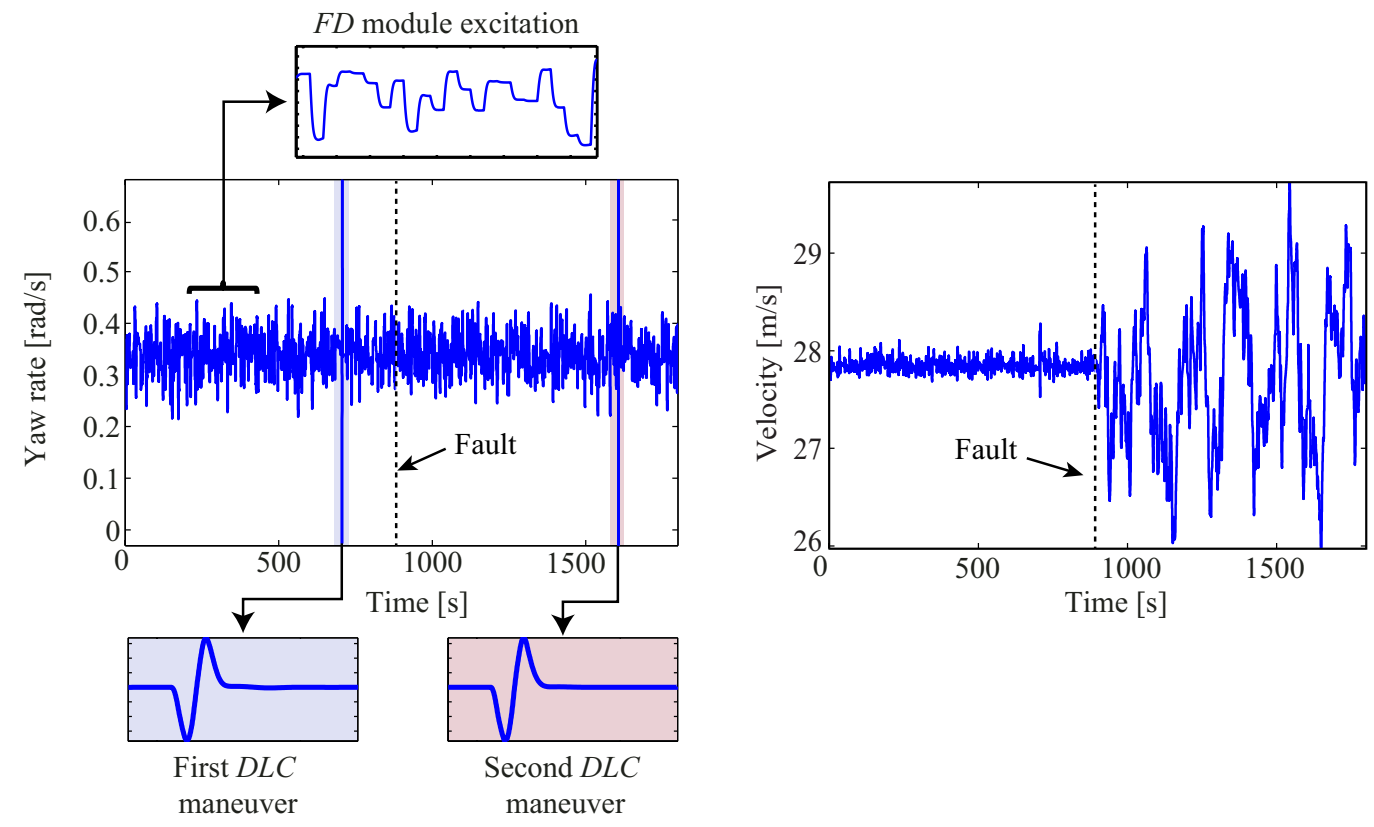

Figure 19: Resulting responses for (left) Yaw rate and (right) forward velocity.

Considering this test and requirements 1-5 the following behavior is required to validate the reliability of the proposed strategy:

- The FD module must detect that the velocity control system is operating without fault and schedule the correct yaw-rate controller.

- The FD must maintain the detection of the healthy state until the fault is introduced.

- The yaw-rate response during the first $D L C$ must be close to that of a neutral steer setting.

- After the fault appears $(t=900 s)$, the $F D$ module must detect the fault and switch to the appropriate yaw-rate controller.

- The FD module must maintain the faulty state during the rest of the test.

- The yaw-rate response during the second $D L C$ must be close to that of a neutral steer setting. 
- Finally, when the velocity control system is healthy the vehicle must maintain the target velocity.

The effectiveness of the FD module is demonstrated in Fig. 20. This figure shows that the false positive condition is quickly eliminated and the fault is adequately detected. In each case the appropriate state is maintained without bouncing due to the commutation of the yaw-rate controller. This shows that the controller commutation is stable and the $F D$ module fulfills requirements $\mathbf{1 - 2}$.

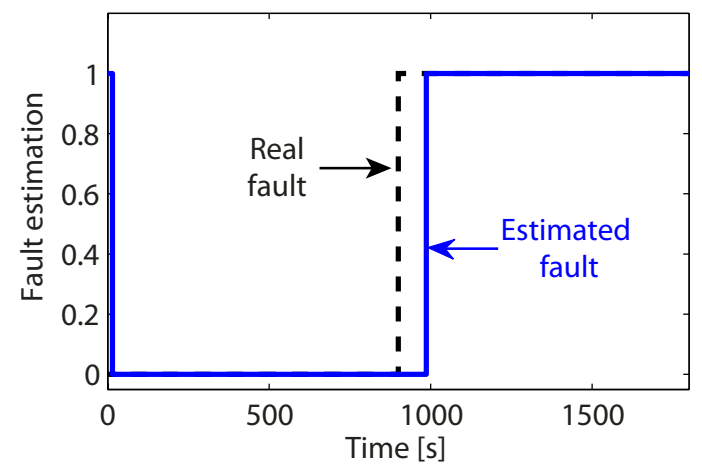

Figure 20: Fault detection during the test.

The performance of the yaw-rate $F T C$ is evaluated with two $D L C$ maneuvers, one before the fault appears and one after. The resulting yaw-rate responses are shown in Fig. 21. In addition, a comparison with the resulting response when the $F T C$ is not used is also presented in the second $D L C$ maneuver. When the fault is not accommodated, the yaw-rate response is clearly affected. The Root Mean Square $(R M S)$ of the yaw rate tracking error during the $D L C$ maneuver increases $35.83 \%$ compared to that of the FTC scheme. These results show that the $F T C$ scheme fulfills requirement 4.

A close-up of the behavior of the vehicle velocity during the $D L C$ maneuvers is shown in Fig. 22. This figure clearly shows that when the VelCS is activated, the vehicle maintains the required velocity and is able to reject the cross-coupling from the steering subsystem to a negligible level, fulfilling requirement 5. Conversely, when the VelCS fails, this variable has a significant amount of variation, as expected.

As mentioned before, the $F D$ module requires excitation of the lateral dynamics. In the test this was achieved by introducing a low amplitude pseudo random stair sequence for the yaw rate reference. A close up of the response 

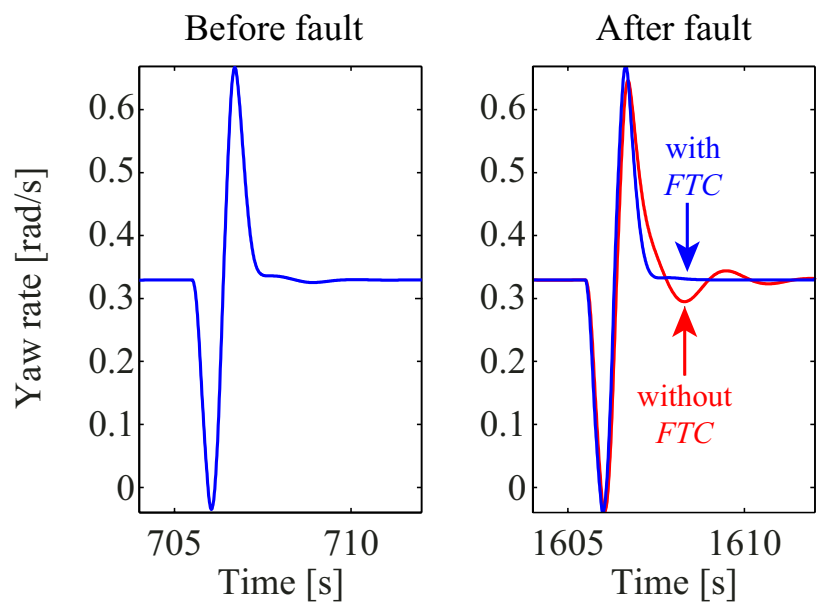

Figure 21: Yaw rate response in the $D L C$ maneuvers.
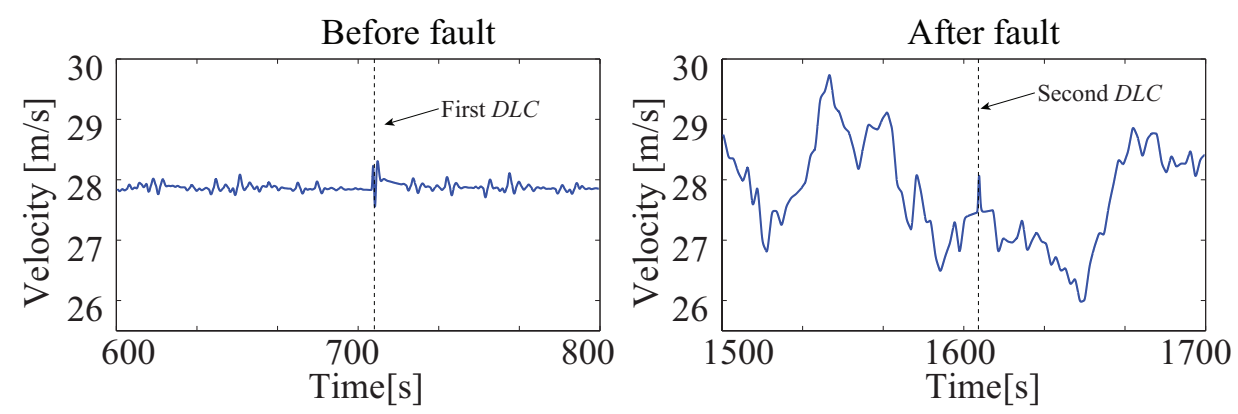

Figure 22: Close-up of the velocity response before and after the fault.

of the yaw rate during the excitation phase is shown in Fig. 23. When the fault is first introduced, before the $F D$ module is able to detect it, there is a clear increment on the overshoot. Later, when the fault is detected, the FTC is able to recover the desired neutral-steer characteristics by reducing the overshoot. This figure also shows that the response is stable when the $F D$ module operates in any detection condition, fulfilling requirement 3.

Finally, the use of a more comprehensive vehicle model allows monitoring several vehicle variables. This can be useful to assess the effects of crosscoupling among vehicle subsystems. As follows some of these variables are presented in Fig. 24.

The first observation is that most variables appear to be highly coupled with the steering subsystem. This cross-coupling can be evaluated by looking 

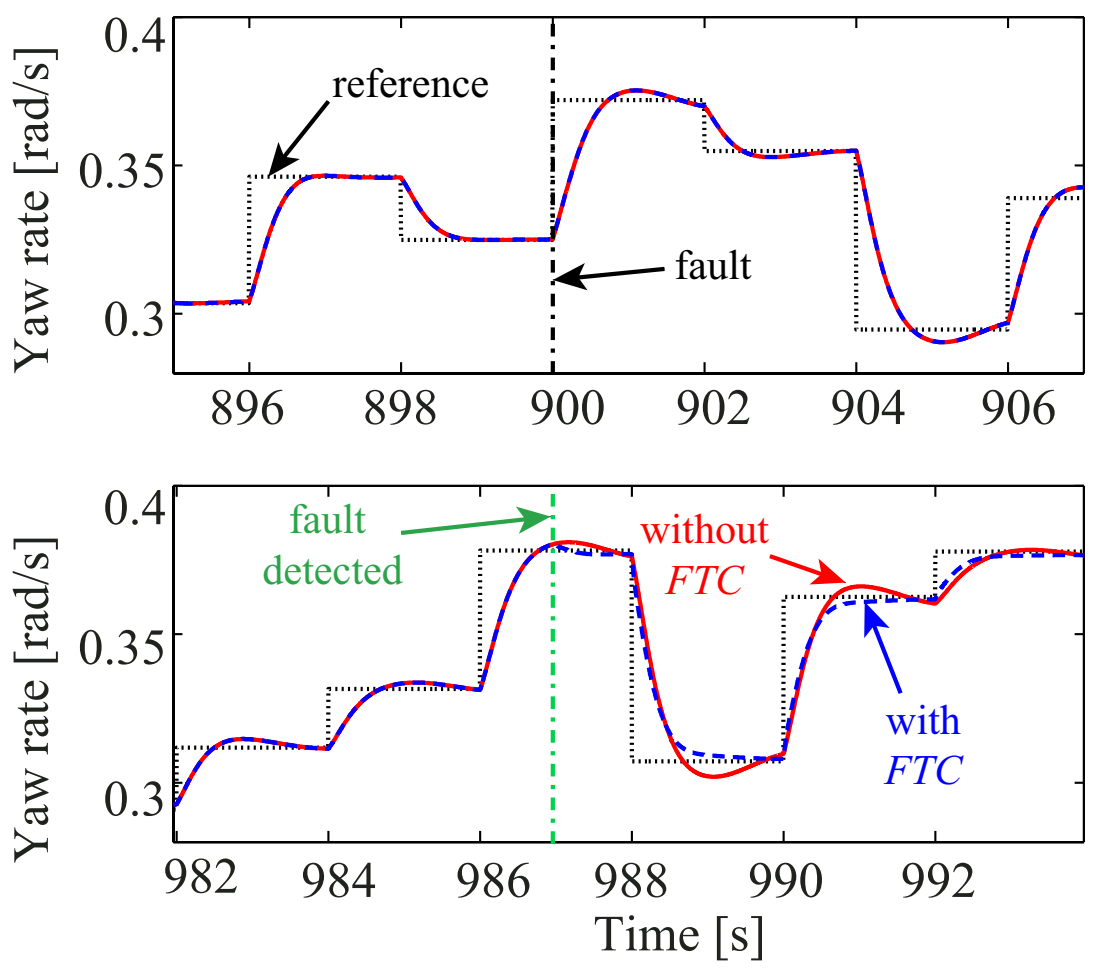

Figure 23: Close up of the yaw rate response at fault and fault detection times.

for peaks at the times of the $D L C$ maneuvers (at $t=705 \mathrm{~s}$ and $t=1605 \mathrm{~s}$ ). The least coupled variables are the longitudinal slip, Fig. 24c, and the tires angular speed, Fig. 24a. The most coupled are the roll angle, Fig. 24e, the pitch angle, Fig. 24f, and the side-slip angles, Fig. 24d.

Another interesting observation is the effect of the fault in the VelCS. While some of these variables are almost unaffected by the failure, others are importantly affected. The least affected are the tires normal force, the sideslip angles and the roll angle. On the other hand before the fault occurrence, the rotational speed of the tires presents a small variation and, since the vehicle is in a cornering condition, the rotational speed of the outer tires $\left(\omega_{2}\right.$ and $\left.\omega_{3}\right)$ is higher than the speed of the inner ones $\left(\omega_{1}\right.$ and $\left.\omega_{4}\right)$. When the fault appears in the $\mathrm{VelCS}$, the variation of the rotational speed increases; in particular, the standard deviation of the rotational speed increases in average $1286 \%$, Fig. 24.a. When the velocity control system is deactivated, the pitch is reduced by $83.16 \%$ since there is no accelerating torque applied to regulate 
a) Tire angular speed

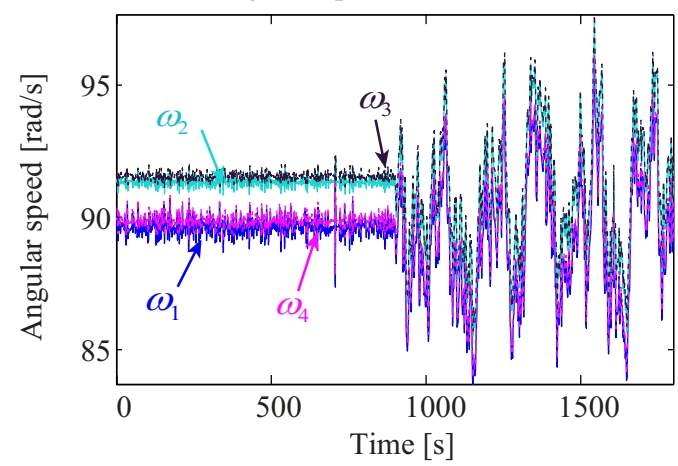

c) Longitudinal slip

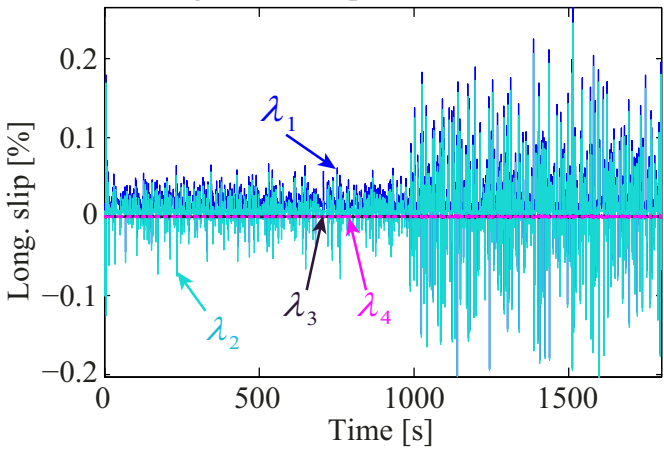

e) Roll

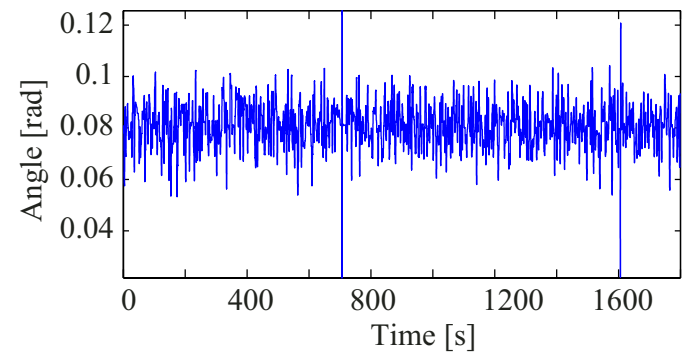

b) Tire normal force

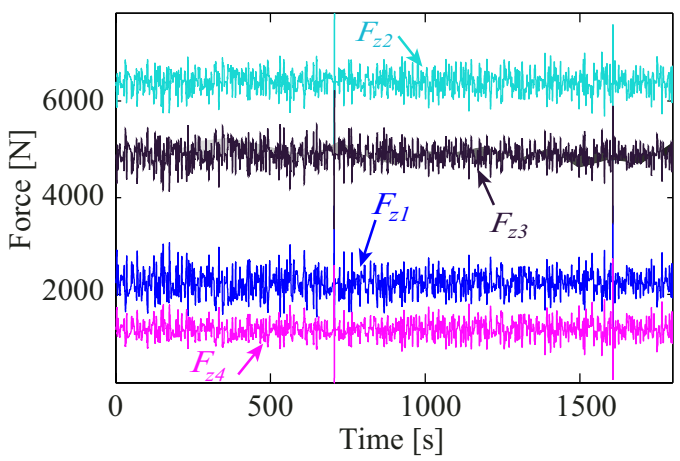

d) Side-slip angle

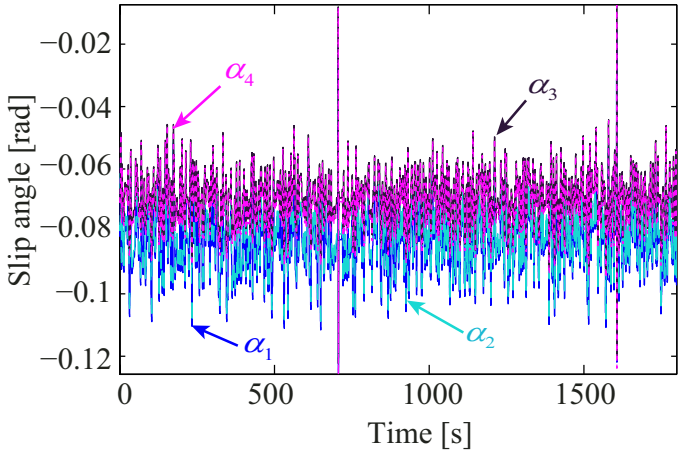

f) Pitch

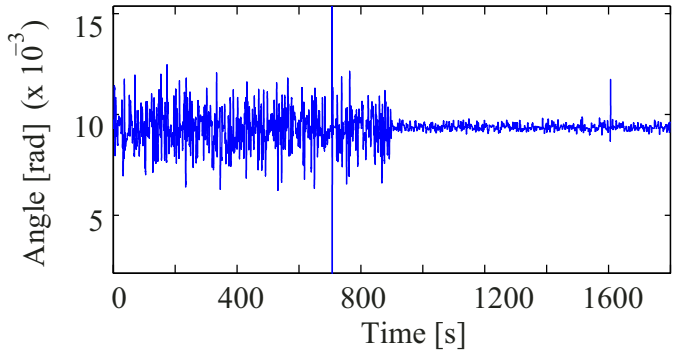

Figure 24: Response of other vehicle variables during the $D L C$ test.

the velocity, Fig. 24.f.

Finally, the previous discussion shows that the vehicle has complex internal cross-coupling dynamics that need to be considered when designing multiple $V C S$. The pitch angle is clearly more coupled with the steering subsystem when the velocity control loop is activated, whereas the contrary occurs in the case of the tires angular velocity. 


\section{Conclusions}

The use of Vehicle Control Systems ( VCS ) allows improving several characteristic of automotive vehicles. Traditionally, the design of these control systems has been achieved by using simplified models that focus in a single vehicle subsystem; that is, using a decentralized control approach. However, the simultaneous use of multiple $V C S$ brings up the problem of subsystem cross-coupling. Although this problem can be solved using a centralized control approach, decentralized control is still attractive due to its simplicity and the possibility of independent operation.

In this article the goal is to design steering and velocity controllers which can operate in a completely decentralized manner, but are also immune to the effects of cross-coupling. In this context, steering controllers are normally designed using the simplified bicycle model. However, it is shown that this model lacks crucial cross-coupling dynamics with the longitudinal subsystem of the vehicle. Therefore, a more complete model considering the four tires, and full body and tire dynamics is proposed.

A further problem with simultaneous $V C S$ operation is the effect of a failure of one subsystem over the other subsystems. While the effects and accommodation of faults within the same subsystem have been thoroughly studied, its effect over other subsystems is still not widely reported. It is shown that failure of the velocity control system may degrade the performance of the steering subsystem. A thorough analysis of the cross-coupling between these variables allowed to design a Fault Tolerant Control (FTC) for the yaw-rate tolerant to faults in the velocity control system. Since independent operation of the steering and velocity controllers is desired, the steering FTC is designed so that no data from the velocity control subsystem is required. In particular, it is shown that it is possible to detect faults in the velocity control system using data of the steering control system by exploiting the cross-coupling between the subsystems. This enables an independent fault tolerant operation.

Finally, it is complex to deal with multiple VCS because fo the complexity of the interactions among subsystems and because of the vastness of conceivable variable pairings and operating conditions. Although, these results represent a particular case, the proposed approach could be relevant to other systems. 


\section{Acknowledgement}

Authors thank CONACyT (PCP project 06/2013 Mexico-France) for their partial support to this research.

\section{References}

[1] Y. Zhang, J. Jiang, Bibliographical Review on Reconfigurable Faulttolerant Control Systems, Annual Reviews in Control 32 (2008) 229252 .

[2] S. X. Ding, S. Schneider, E. L. Ding, A. Rehm, Fault Tolerant Monitoring of Vehicle Lateral Dynamics Stabilization Systems, in: Proceedings of the $44^{\text {th }}$ IEEE Conf on Decision and Control, 2005, pp. 2000-2005.

[3] T. Hsiao, M. Tomizuka, Sensor Fault Detection in Vehicle Lateral Control Systems via Switching Kalman Filtering, in: Proc of the 2005 American Control Conf, 2005, pp. 5009-5014 vol. 7.

[4] L. M. Ho, D. Ossmann, Fault Detection and Isolation of Vehicle Dynamics Sensors and Actuators for an Overactuated X-by-Wire Vehicle, in: 53th IEEE Conf on Decision and Control, 2014, pp. 6560-6566.

[5] S. Aouaouda, M. Chadli, O. Bouhali, Observer-based Fault Tolerant Tracking Control for Vehicule Lateral Dynamics, in: 2013 Int Conf on Control, Decision and Information Technologies, 2013, pp. 051-056.

[6] S. Aouaouda, M. Chadli, H. R. Karimi, Robust Static Output-Feedback Controller Design against Sensor Failure for Vehicle Dynamics, IET Control Theory Applications 8 (9) (2014) 728-737.

[7] M. Oudghiri, M. Chadli, A. Elhajjaji, Observer-based Fault Tolerant Control for Vehicle Lateral Dynamics, in: European Control Conf, 2007, pp. 632-637.

[8] M. Oudghiri, M. Chadli, A. E. Hajjaji, A Fuzzy Approach for Sensor Fault-Tolerant Control of Vehicle Lateral Dynamics, in: 2007 IEEE Intl Conf on Control Applications, 2007, pp. 1221-1226.

[9] Y. D. Song, H. N. Chen, D. Y. Li, Virtual-Point-Based Fault-Tolerant Lateral and Longitudinal Control of 4W-Steering Vehicles, IEEE Trans on Intelligent Transportation Systems 12 (4) (2011) 1343-1351. 
[10] J. Huang, M. Tomizuka, LTV Controller Design for Vehicle Lateral Control under Fault in Rear Sensors, IEEE/ASME Trans on Mechatronics 10 (1) (2005) 1-7.

[11] S. Varrier, D. Koenig, J. J. Martinez, Robust Fault Detection for Vehicle Lateral Dynamics, in: 51 ${ }^{\text {th }}$ IEEE Conf on Decision and Control, 2012, pp. 4366-4371.

[12] S. Varrier, D. Koenig, J. J. Martinez, Robust Fault Detection for Uncertain Unknown Inputs LPV System, Control Engineering Practice 22 (2014) $125-134$.

[13] J. Andreasson, T. Bünte, Global Chassis Control based on Inverse Vehicle Dynamics Models, Vehicle System Dynamics 44 (sup1) (2006) 321328.

[14] P. Falcone, F. Borrelli, J. Asgari, H. Tseng, D. Hrovat, A Model Predictive Control Approach for Combined Braking and Steering in Autonomous Vehicles, in: Mediterranean Conf. on Control Automation, Athens, Greece, 2007, pp. 1-6.

[15] M. Aripin, S. Yahaya, K. Danapalasingam, K. Peng, N. Hamzah, M. Ismail, A Review of Active Yaw Control System for Vehicle Handling and Stability Enhancement, Int J of Vehicular Technology 2014 (2014) 1-15.

[16] M. Valasek, O. Vaculin, J. Kejval, Global Chassis Control: Integration Synergy of Brake and Suspension Control for Active Safety, in: $7^{\text {th }}$ Int Symp on Advanced Vehicle Control, Netherlands, 2004, pp. 495-500.

[17] P. Gáspár, Z. Szabó, J. Bokor, Lpv design of fault-tolerant control for road vehicles, Int J of Applied Mathematics and Computer Science 22 (1) (2012) 173-182.

[18] R. Jazar, Vehicle Dynamics: Theory and Application, $2^{\text {nd }}$ Edition, Springer New York, 2013.

[19] H. B. Pacejka, Chapter 1 - Tire Characteristics and Vehicle Handling and Stability, in: H. B. Pacejka (Ed.), Tire and Vehicle Dynamics, $3^{\text {rd }}$ Edition, Butterworth-Heinemann, Oxford, 2012, pp. 1 - 58. 
[20] L. Guzzella, C. Onder, Introduction to Modeling and Control of Internal Combustion Engine Systems, Springer Berlin Heidelberg, 2009.

[21] J. O'Reilly, W. E. Leithead, Multivariable Control by Individual Channel Design, Int J of Control 54 (1) (1991) 1-46.

[22] R. Rajamani, Vehicle Dynamics and Control, Mechanical Eng Series, Springer US, 2011.

[23] C. Poussot-Vassal, O. Sename, L. Dugard, A LPV $/ H_{\infty}$ Global Chassis Controller for Handling Improvements involving Braking and Steering Systems, in: $47^{\text {th }}$ IEEE Conf on Decision and Control, Cancun, México, 2008, pp. 5366-5371.

[24] M. Doumiati, O. Sename, L. Dugard, J.-J. Martinez-Molina, P. Gaspar, Z. Szabo, Integrated Vehicle Dynamics Control via Coordination of Active Front Steering and Rear Braking, European J of Control 19 (3) (2013) 121-143.

[25] M. Cook, Flight Dynamics Principles: A Linear Systems Approach to Aircraft Stability and Control, Aerospace Eng, Butterworth-Heinemann, 2013.

[26] C. A. Vivas-Lopez, J. C. Tudon-Martinez, D. Hernandez-Alcantara, R. Morales-Menendez, Global Chassis Control System Using Suspension, Steering, and Braking Subsystems, Mathematical Problems in Engineering (263424) (2015) 18. 